\title{
Reviewing Potentially Inappropriate Medication in Hospitalized Patients Over 65 Using Explicit Criteria: A Systematic Literature Review
}

\section{Hesah Alshammari (D) \\ Eman Al-Saeed \\ Zamzam Ahmed \\ Zoe Aslanpour}

Department of Clinical and Pharmaceutical Sciences, School of Life and Medical Sciences, University of Hertfordshire, Hatfield, UK
Correspondence: Hesah Alshammari Email h.hesah-a-j-m-a-alshammari@herts.ac. uk

\begin{abstract}
Potentially inappropriate medication (PIM) is a primary health concern affecting the quality of life of patients over 65. PIM is associated with adverse drug reactions including falls, increased healthcare costs, health services utilization and hospital admissions. Various strategies, clinical guidelines and tools (explicit and implicit) have been developed to tackle this health concern. Despite these efforts, evidence still indicates a high prevalence of PIM in the older adult population. This systematic review explored the practice of using explicit tools to review PIM in hospitalized patients and examined the outcomes of PIM reduction. A literature search was conducted in several databases from their inception to 2019. Original studies that had an interventional element using explicit criteria detecting PIM in hospitalized patients over 65 were included. Descriptive narrative synthesis was used to analyze the included studies. The literature search yielded 6116 articles; 25 quantitative studies were included in this systematic literature review. Twenty were prospective studies and five were retrospective. Approximately, 15,500 patients were included in the review. Various healthcare professionals were involved in reviewing PIM including physicians and hospital pharmacists. Several tools were used to review PIM for hospitalized patients over 65 , most frequently Beer's criteria and the STOPP/START tool. The reduction of PIM ranged from $3.5 \%$ up to $87 \%$. The most common PIM were benzodiazepines and antipsychotics. This systematic review showed promising outcomes in terms of improving patient outcomes. However, the reduction of PIM varied in the studies, raising the question of the variance between hospitals in the explicit tools used for review. Additional studies need to be conducted to further investigate the outcomes of reviewing PIM at different levels, as well as assessing the cost-effectiveness of using explicit tools in reducing PIM.
\end{abstract}

Keywords: older population, adverse drug effects, drug review tools, PIM

\section{Introduction}

Potentially inappropriate medication (PIM) is a health concern that highly affects the quality of life for patients 65 years and over. Older patients who were prescribed such medication have experienced an increase in falls, adverse drug reactions, ${ }^{1,2}$ healthcare costs, health services utilization and hospital admissions. ${ }^{3,4}$ PIM are defined as medications for which the risk outweighs the potential benefits. ${ }^{5}$

Many interventions have been developed to tackle PIM. As part of the daily routine in hospitals, physicians, pharmacists, and other healthcare professionals (HCP) have assumed the responsibility to reduce PIM in patients over 65. An observational study was conducted in France for 6 months to evaluate the routine care provided in a geriatric unit. ${ }^{6}$ The study confirmed that the usual care included 
medication reviews done by geriatricians. This resulted in 275 medication changes, with 158 medications stopped, 53 medications replaced, and 64 new medications initiated. Notably, 132 (61.11\% (95\% CI = [54.61-67.61]) patients over 65 had at least one medication discontinued during their hospital stay. This study reflected the practice of optimizing older patients' medication as part of routine care; however, the Hawthorne effect might have influenced the physician-patient communication. ${ }^{7}$ The changes in PIM prevalence during the hospital stay may indicate that HCP interventions contributed to PIM reduction. This was concluded by Laroche et al in a prospective study after the HCPs reduced PIMs by $22.4 \%$ during the older patients' hospital stay. ${ }^{8}$ To further support that, a UK-based retrospective study was conducted in an acute hospital in England examining 195 patients over 65, ${ }^{9}$ which revealed that the prevalence of PIM on admission was $26.7 \%$ with 74 PIMs detected in 52 patients; at discharge, the prevalence of PIM was $22.6 \%$ with 51 PIMs detected in 44 patients. A statistically significant change in PIM prevalence was found between PIM on admission and discharge. Additionally, the study disclosed that a small number of patients received a follow-up letter when prescribed a PIM. Similar findings were observed by Komagamine in his retrospective study, based on a hospital database in Japan, which concluded that the number of PIM upon discharge was fewer than the number of PIM on admission, indicating a significant reduction rate $(0.48$ on admission vs 0.53 at discharge). ${ }^{10}$

An overview of systematic reviews that investigated interventions aimed at PIM reduction found that several interventions were employed to reduce PIM. These included medication review services, pharmaceutical intervention, computerized systems and educational interventions. ${ }^{11}$ The studies included in the overview were conducted in various health care settings such as hospitals, primary care clinics, nursing homes and longterm care facilities.

The tools to detect PIM can be categorized as implicit (judgment based), explicit (criteria based) or combined (both judgment and criteria based). Implicit tools contain questions that are designed to examine the effectiveness and safety of each medication such as the Medication Appropriateness Index (MAI). Explicit tools comprise a list of medications that are known, based on evidence, to be inappropriately prescribed to older patients. Examples of explicit tools are the Beers Criteria and the STOPP/ START tool (Screening Tool of Older Persons'
Prescriptions/Screening Tool to Alert to Right Treatment). Clinical expertise is needed to apply the tools with recommendations tailored to each patient. ${ }^{12}$

The Beers Criteria was produced in 1991 through a Delphi technique of 13 experts. ${ }^{13}$ The Beers criteria was recently update in 2019 through the Delphi method of 13 experts (physicians, pharmacist, and nurses) who have already contributed to Beers criteria 2015 update. A literature search in both PubMed and Cochrane Library was conducted to identify relevant literature. The literature search yielded 67 systematic reviews and/or meta-analyses, 29 controlled clinical trials and 281 observational studies. This evidence went under review in a cycle of evaluation by the expert panel. Evidence evaluation was done through two approaches: the American College of Physicians (ACP) and the Grading of Recommendations Assessment, Development and Evaluation guidelines for clinical practice guideline development (GRADE). There were two criteria to assess the evidence which are quality of evidence (high-moderate-low) and strength of recommendation (strong or weak).

The STOPP/START tool was developed in 2008 and produced an update in 2015. ${ }^{14}$ The recent update was based on a literature search in three databases (PubMed, Embase and Cochrane Library) to find systematic reviews, randomised controlled trials and reviews. In addition to the literature search, British National Formulary (BNF) the National Institute for Health and Care Excellence (NICE) and the Scottish Intercollegiate Guidelines Network (SIGN) to search references of guidelines as well as recent published textbooks. The selected articles were categorized into the physiological systems after being assessed by the three members of the research team for their suitability as an evidence to be presented to the expert panel of 19 experts from 13 European countries. The expert panel was not asked to read the full articles nor assess the evidence through standardised rating; however, abstracts of the evidence was presented, and reference bank was supplied to access full articles if needed. To enable online Delphi panel, SurveyMonkey ${ }^{\circledR}$ was utilized to achieved consensus through 5-point Likert scale $(0=$ do not know; $1=$ strongly agree; $2=$ agree; $3=$ neutral; $4=$ disagree; $5=$ strongly disagree). It is worth to note that the expert panel were initially asked to comment on the 2008 version of STOPP/START and to reflect on its validity and relevance.

Although the literature suggests that PIM are identified through explicit tools during a patient's hospital stay, there is a paucity of data as to when these explicit tools are used 
Table I Population, Intervention, Outcome

\begin{tabular}{|l|l|}
\hline Population & Hospitalized 65 Years and Over Patients \\
\hline $\begin{array}{l}\text { Intervention } \\
\text { Outcome }\end{array}$ & $\begin{array}{l}\text { Explicit tool application } \\
\text { PIM reduction (primary outcome) and clinical and non-clinical outcomes (secondary outcomes) }\end{array}$ \\
\hline
\end{tabular}

within a hospital setting. This systematic review aimed to explore the practice of reviewing PIM in hospitalized patients over 65 , using explicit tools. The objectives were:

- To explore the PIM review process in terms of the explicit tools used, HCP involved, stage of hospitalization and resources utilized.

- To identify the common PIM and their clinical relevance in hospitals.

- To investigate the clinical and non-clinical outcomes of the PIM review.

\section{Method}

A systematic literature search was carried out from February 9 to February 13, 2019, using predefined search terms. The literature search was updated on 20th of April 2021. This review was registered in PROSPERO under the registration number CRD42019131104.

Based on the research question "how do healthcare professionals review PIM in hospitalized 65 years and over patients using explicit tools and what are the outcomes of the review process?", the PIO format is shown in Table 1.

The search terms were obtained from concepts of the research question, keywords of relevant articles and the search strategy of systematic reviews. ${ }^{15}$ The search terms were validated by the research team and an information manager. The search terms were piloted in PubMed and relevant data were found. ${ }^{16}$ The search term combination is detailed in Table 2. The search was restricted to English articles only; no filter was used to limit the studies in the selected period. The following databases were searched: PubMed, Scopus, PsycINFO, CINHAL plus, Web of Science, all Ovid journals and OpenGrey. All the results were exported to EndNote 9 as a reference manager and to eliminate duplication.

\section{Selection}

A set of predefined inclusion and exclusion criteria were constructed to answer the aim and objectives of this review (refer to Table 3). Two reviewers completed the title and abstract screening. Any disagreement was resolved by a discussion, then an agreed decision to include or exclude was reached.

\section{Data Extraction}

The data extraction form was developed (Appendix 1) and Microsoft Excel was used to extract and tabulate the data of the included studies. Two reviewers conducted the data extraction and extracted the following information:

1. Author and year of publication

2. Country and setting of the study

3. Study design

4. Number and characteristics of participant

5. Explicit tool used and applied by whom

6. Sources of data used to assist the decision

7. Primary and secondary outcomes (clinical and nonclinical outcomes)

\section{Analysis}

The review included all relevant data without limitations to specific study design, thereby including various types of quantitative studies.

The included articles were narratively synthesized. Narrative synthesis answers research questions that are about the effect intervention and the implementation of the intervention. A description of the included studies contained country, sample, tool used, number of PIM before and after tool application, and additional relevant results.

In this study, the latest version of the Mixed Method Appraisal Tool (MMAT) ${ }^{17}$ was used to evaluate the quality of the included studies. The first version of the MMAT in 2006 was piloted and went through interrater reliability testing. ${ }^{18}$ It was revised in 2011 after being piloted in workshops, which led to the addition of new criteria to assess nonrandomised studies. Version 2018 of the MMAT was subject to content validity and usefulness. In the recent update, usefulness testing through interviews with 20 previous users was conducted to further improve the appraisal tool. In addition, a modified e-Delphi was conducted with 73 experts in the fields different research methods as well as literature reviewer on critical appraisal tool. 
Table 2 The Keywords Combination Used in Each Database for the Systematic Literature Review

\begin{tabular}{|c|c|}
\hline Database & Search Term \\
\hline PubMed & 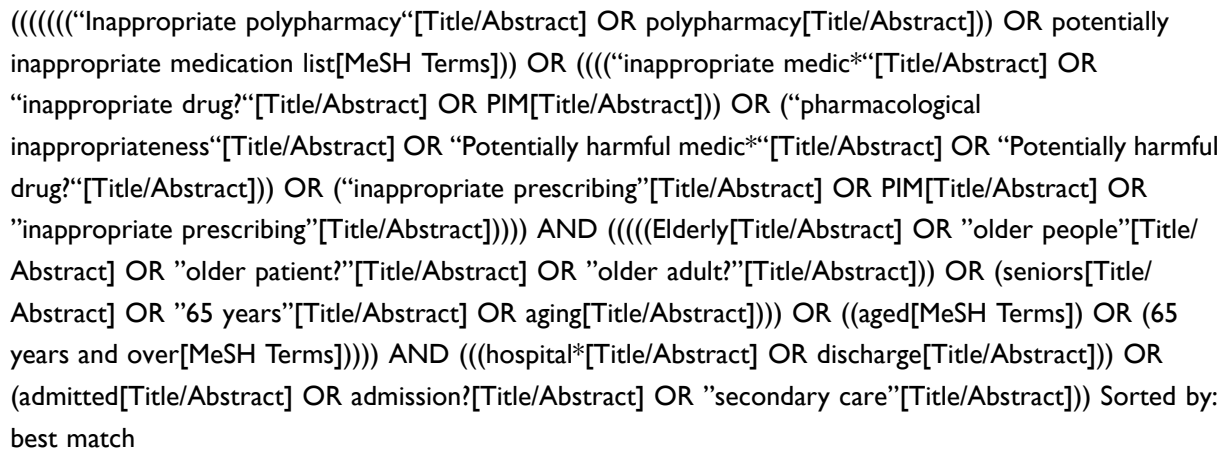 \\
\hline Scopus & 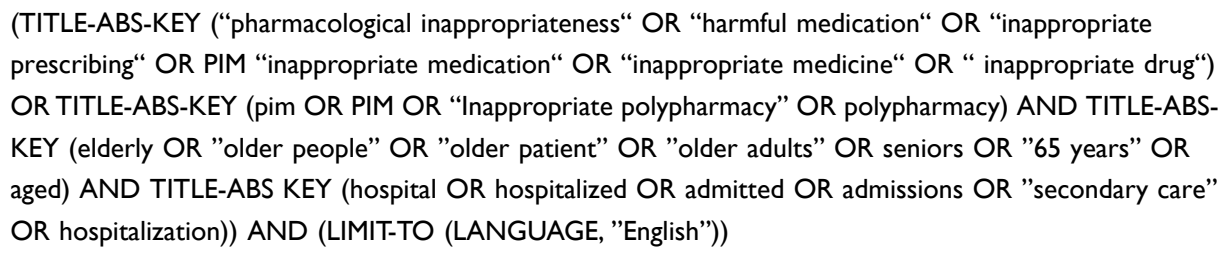 \\
\hline CINHAL PLUS & $\begin{array}{l}\text { AB (“"pharmacological inappropriateness" OR "Potentially harmful medication“ OR "inappropriate } \\
\text { prescribing” OR PIM 'inappropriate medication' OR 'inappropriate medicine' OR 'inappropriate drug' OR } \\
\text { PIM OR 'Inappropriate polypharmacy' OR Polypharmacy) AND AB (Elderly OR older people OR older } \\
\text { adults OR seniors, } 65 \text { years and over OR aged) AND AB (Hospital OR hospitalized OR admitted OR } \\
\text { admissions OR "secondary care" OR hospitalization OR hospitalisation OR hospitalised) }\end{array}$ \\
\hline $\begin{array}{l}\text { All OVID journals, PsycINFO and } \\
\text { Web of Science }\end{array}$ & $\begin{array}{l}\text { (("Inappropriate medic*“ or "inappropriate drug" or "pharmacological inappropriateness" or "inappropriate } \\
\text { prescribing" or "inappropriate polypharmacy" or polypharmacy or PIM or PIM) and (elderly or } 65 \text { years or } \\
\text { age* or "older people" or "older adults" or "older patient" or seniors) and (hospital* or admission or } \\
\text { admitted or discharge or "secondary care")).ab. }\end{array}$ \\
\hline OpenGrey & $\begin{array}{l}\text { "Inappropriate polypharmacy" OR polypharmacy OR "inappropriate medic*“ OR "inappropriate drug*“ OR } \\
\text { PIM OR "pharmacological inappropriateness" OR "Potentially harmful medic*“ OR "Potentially harmful } \\
\text { drug*“ OR PIM OR "inappropriate prescrib* AND elderly OR "older people" OR "older adult*” OR "older } \\
\text { patient*” OR senior* OR "65 years" OR aged AND admissions OR "secondary care" OR Hospital* OR } \\
\text { hospitali* OR admitted lang:"en }\end{array}$ \\
\hline
\end{tabular}

Table 3 List of Inclusion and Exclusion Criteria Applied to the Resulting Articles

\begin{tabular}{|l|l|}
\hline Inclusion Criteria & Exclusion Criteria \\
\hline $\begin{array}{l}\text { - The included study can be either a qualitative or quantitative } \\
\text { original study }\end{array}$ & $\begin{array}{l}\text { - Studies conducted in nursing homes, emergency department and primary } \\
\text { care were excluded. }\end{array}$ \\
- Studies included should be focused on patients 65 years and over & $\begin{array}{l}\text { - Studies focused on terminal illness or end of life patients were excluded. } \\
\text { - Non- English studies were excluded. }\end{array}$ \\
$\begin{array}{l}\text { inappropriate medications } \\
\text { - The included studies should be conducted in a hospital setting }\end{array}$ & \\
\hline
\end{tabular}

An adjacent score was presented in this study to reflect the quality of the study. Two reviewers from the research team conducted the quality assessment. Any disagreement was discussed and the final decision was reported.

\section{Results}

The original literature search yielded 6116 articles and the updated literature yielded 1954 articles. The PRISMA flow diagram below describes the screening process used as shown in Figure 1. The included articles are summarised in Table 4. 


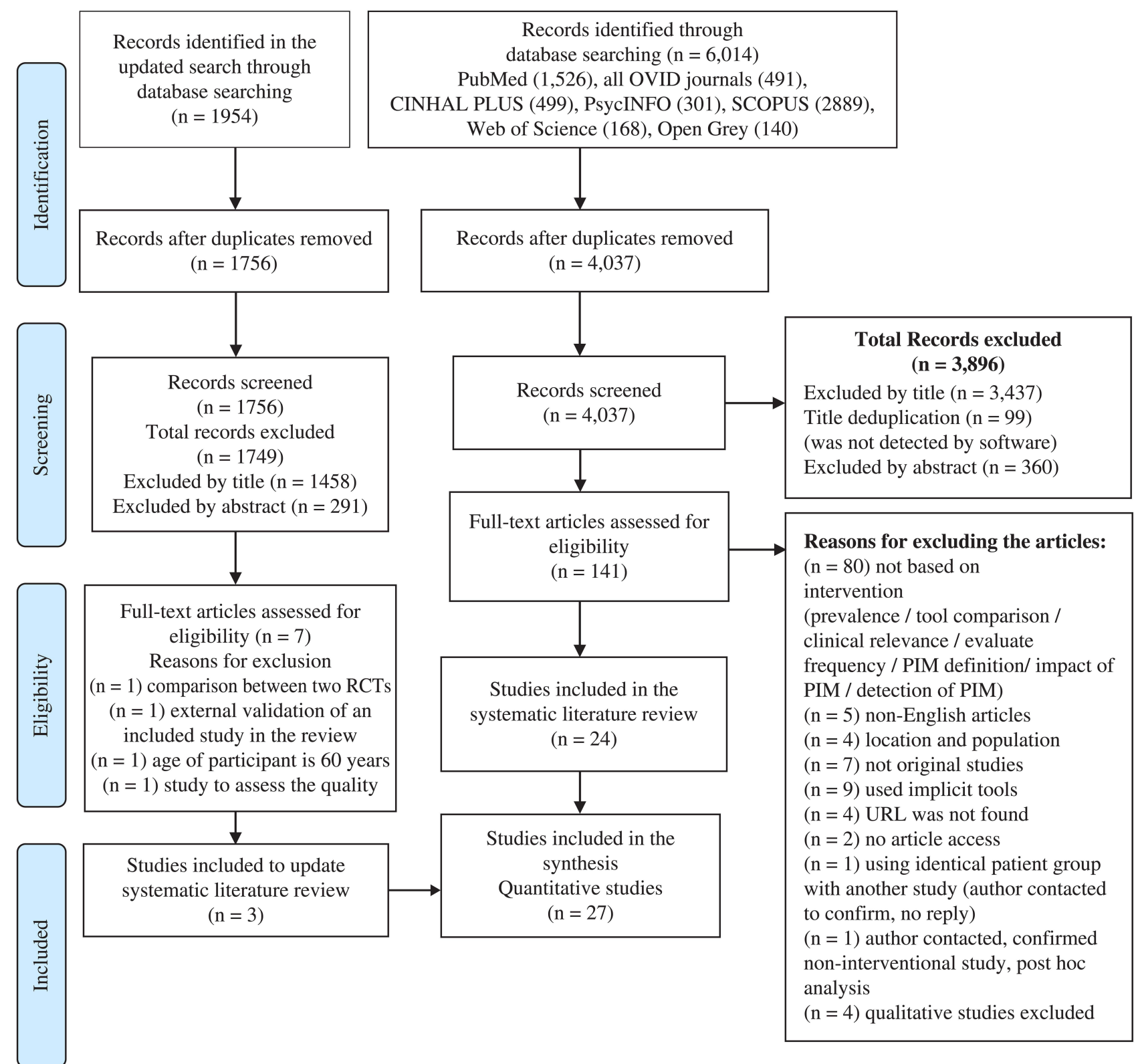

Figure I Results and screening process according to PRISMA guidelines.

Note: Adapted from Page MJ, McKenzie JE, Bossuyt PM, Boutron I, Hoffmann TC, Mulrow CD, et al. The PRISMA 2020 statement: an updated guideline for reporting systematic reviews. BMJ. 2021 Mar 29;372:n71. ${ }^{19}$

\section{Study Characteristics}

Twenty-seven studies were included in this systematic literature review, all of which were quantitative (Table 3). Most quantitative studies were prospective studies, ${ }^{20-42}$ with five retrospective studies. ${ }^{43-47}$ The prospective studies included six randomized controlled trials, ${ }^{20-25}$ one non-randomized controlled trial, ${ }^{26}$ and one ambispective non-randomized controlled study. ${ }^{27}$ Moreover, there was one pilot study, ${ }^{36}$ three before-and-after studies, ${ }^{28-30}$ four observational studies, ${ }^{37,38-42}$ and nine interventional studies. ${ }^{31-35,38,39}$.
Approximately 16,093 patients were included in the studies. Two papers did not state the number of participants.

\section{Explicit Tools}

A wide range of tools was implemented to review PIM in hospitalized older patients. Most studies used various versions of the STOPP/START tool, which was originally developed in Ireland. ${ }^{21,22,27,29,33,37,39,47}$ This tool was used in studies conducted in Ireland, Belgium, India, Switzerland, Spain, and France. Beers criteria were 


\begin{tabular}{|c|c|c|}
\hline 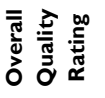 & $\sigma$ & in \\
\hline 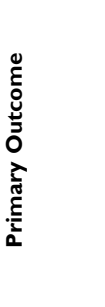 & 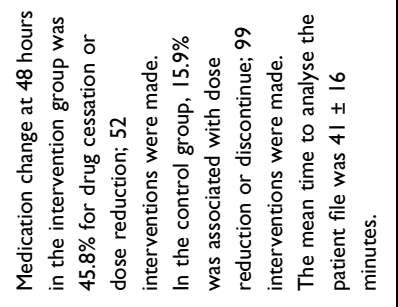 & 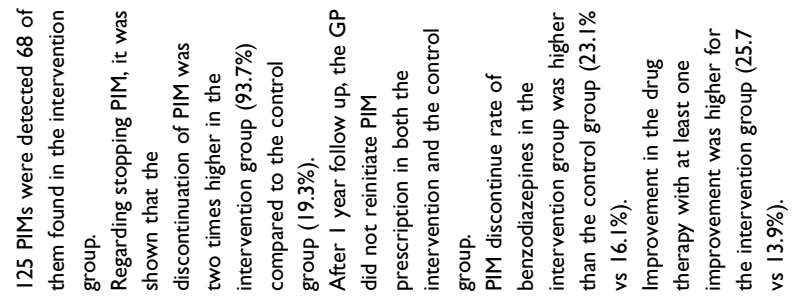 \\
\hline 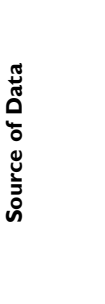 & 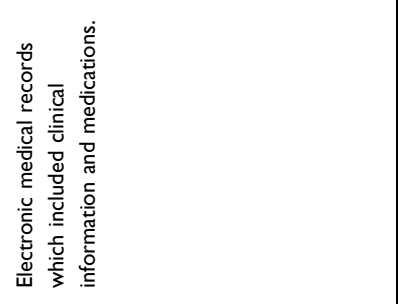 & 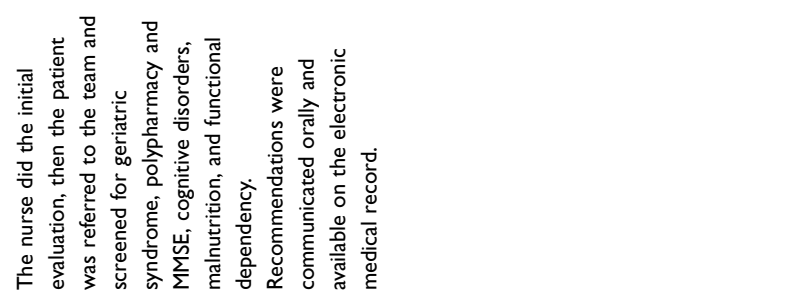 \\
\hline 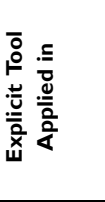 & 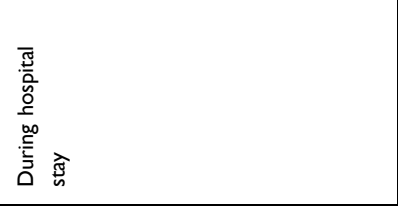 & 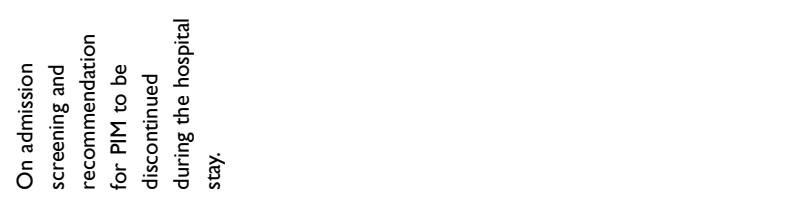 \\
\hline 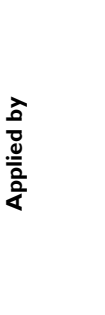 & 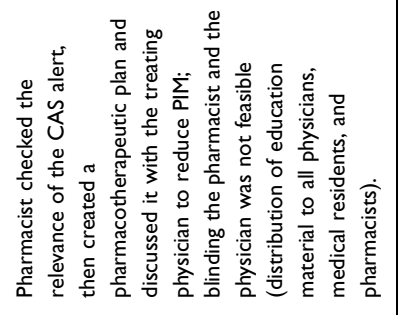 & 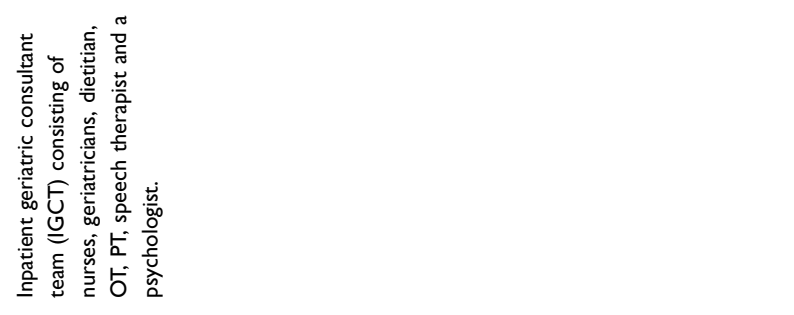 \\
\hline 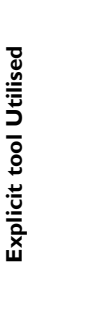 & 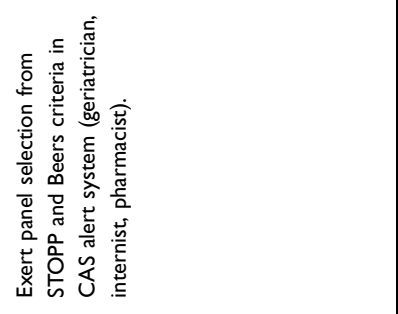 & 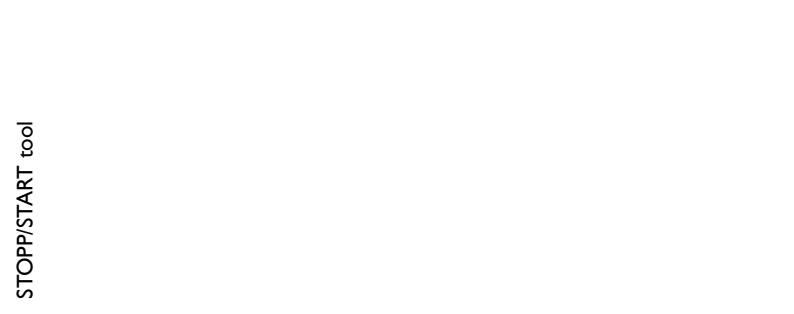 \\
\hline 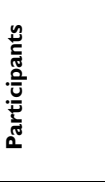 & 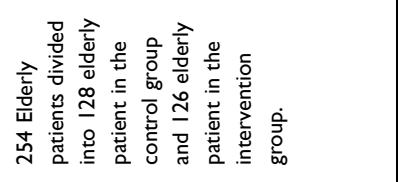 & 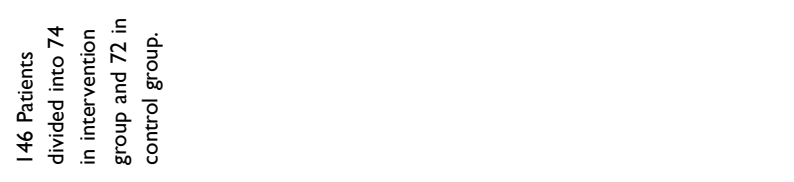 \\
\hline 不总 & 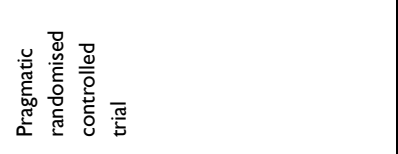 & 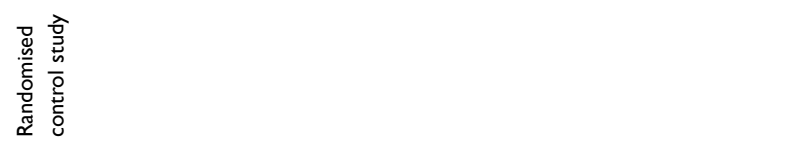 \\
\hline نे & 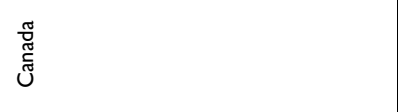 & 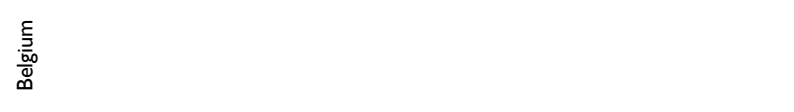 \\
\hline 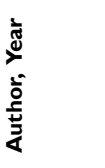 & 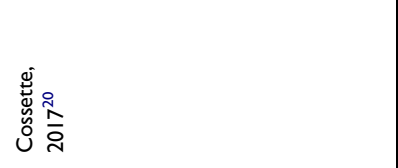 & 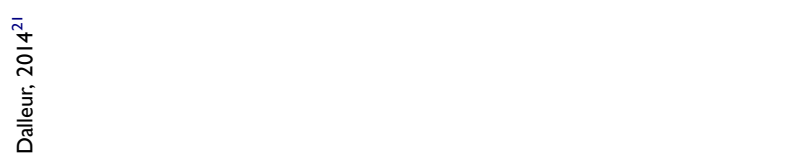 \\
\hline
\end{tabular}




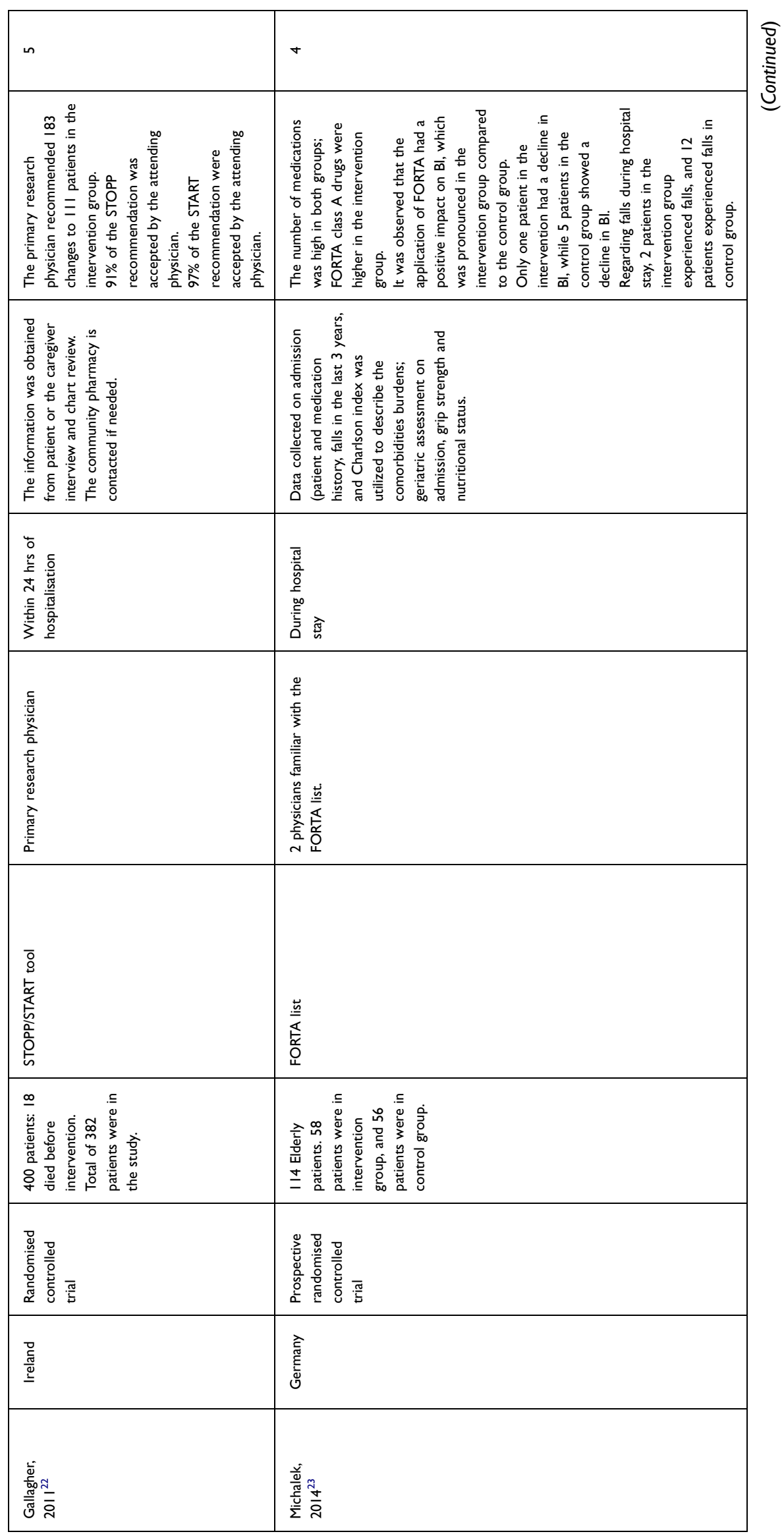




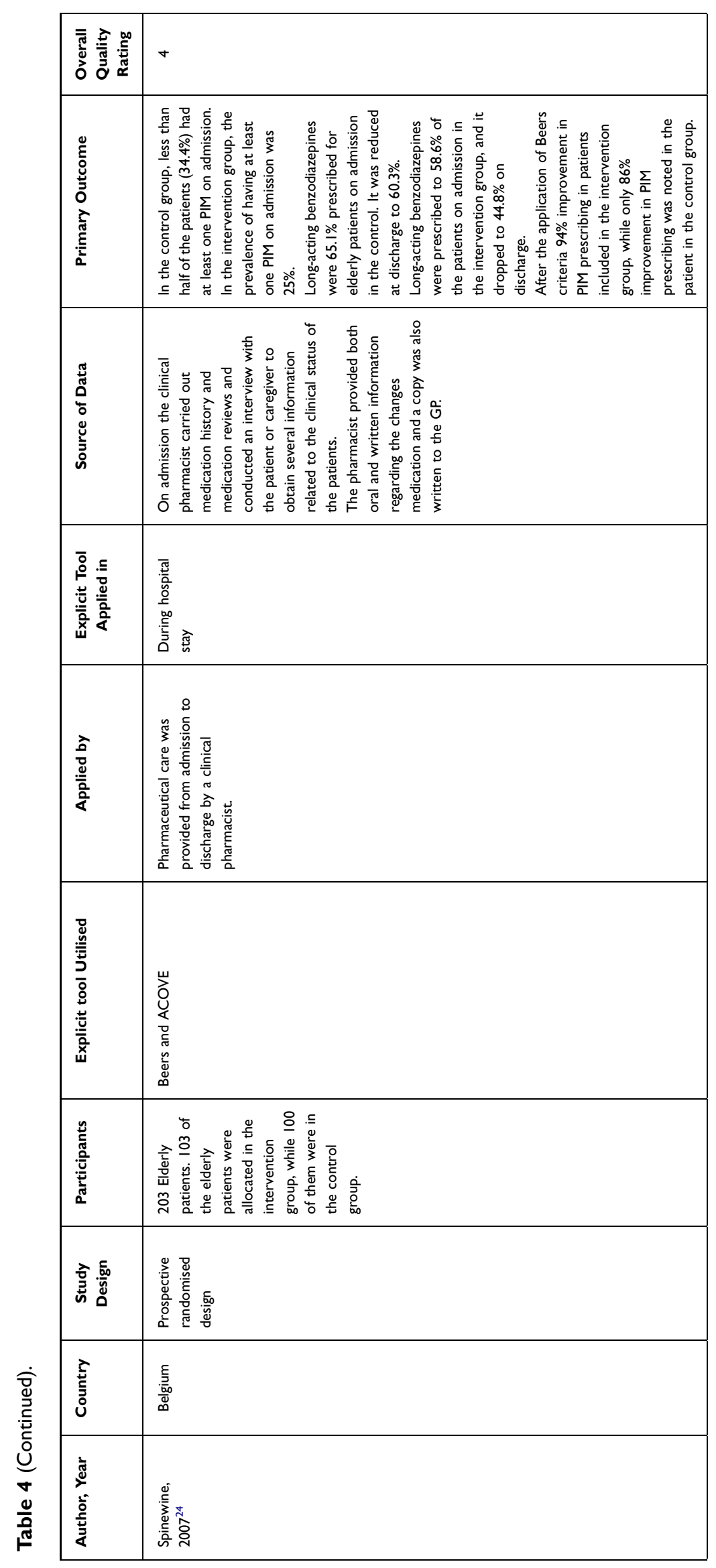




\begin{tabular}{|c|c|}
\hline$\sigma$ & $\sigma$ \\
\hline 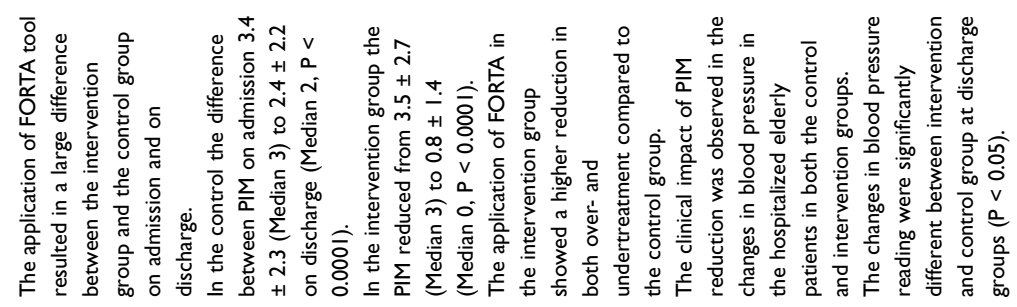 & 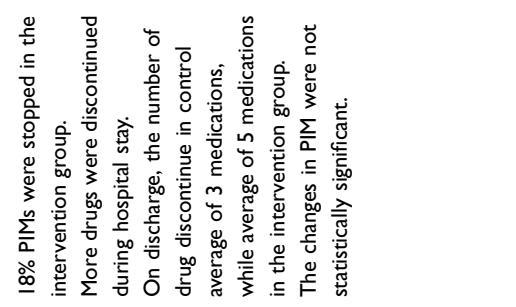 \\
\hline 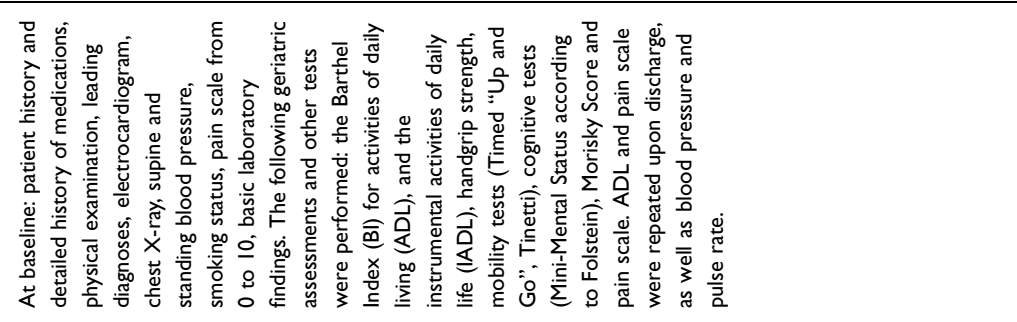 & 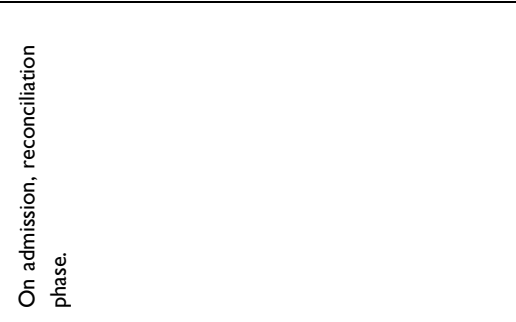 \\
\hline 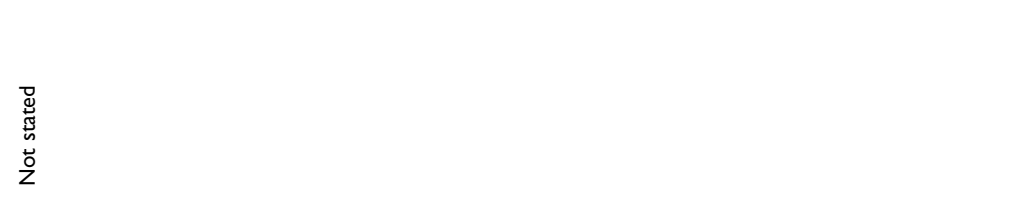 & 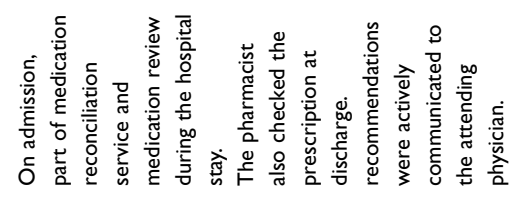 \\
\hline 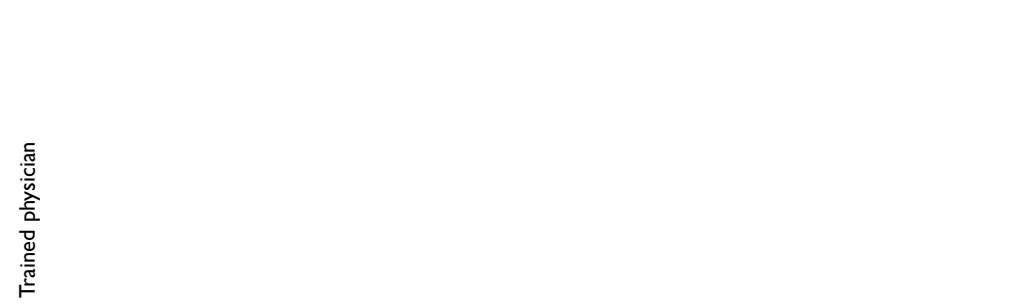 & 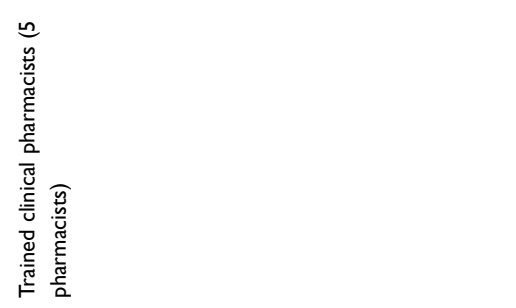 \\
\hline 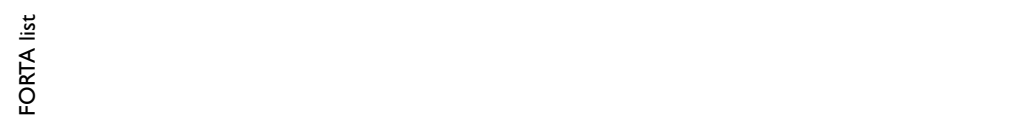 & $\begin{array}{l}\frac{\underline{\underline{\underline{\underline{w}}}}}{0} \\
\underline{\underline{\underline{s}}} \\
\end{array}$ \\
\hline 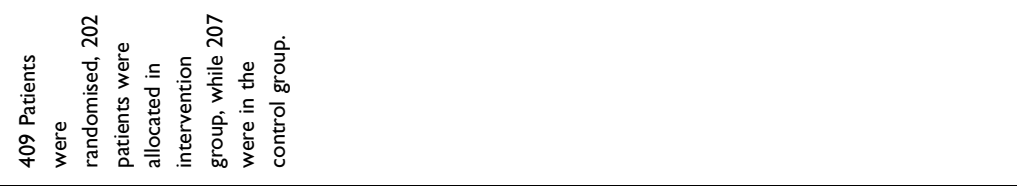 & 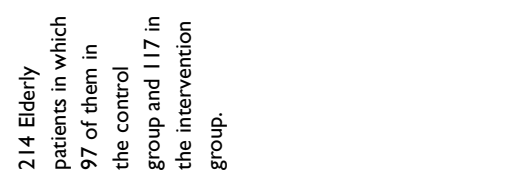 \\
\hline 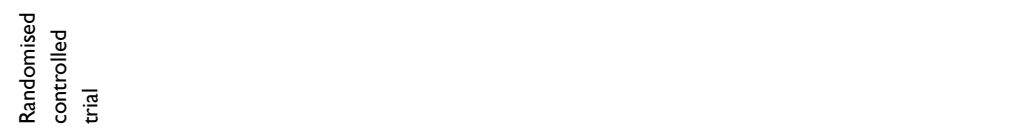 & 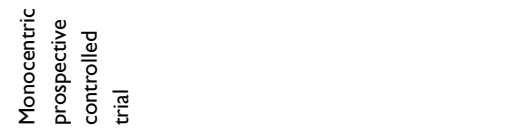 \\
\hline 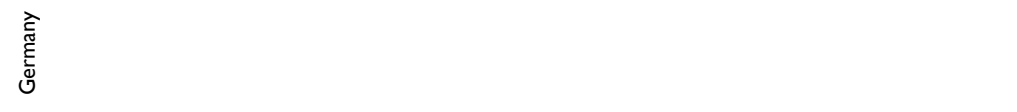 & 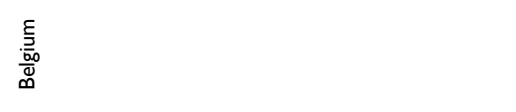 \\
\hline 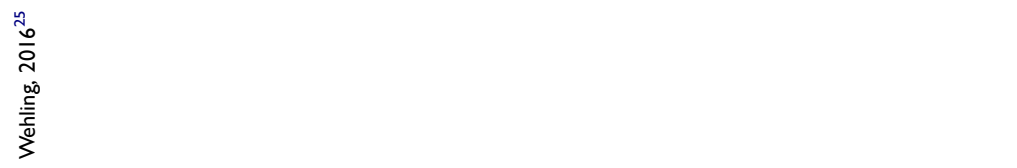 & 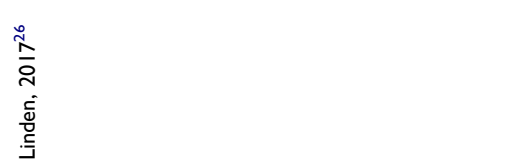 \\
\hline
\end{tabular}




\begin{tabular}{|c|c|c|c|}
\hline 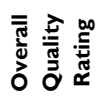 & + & $m$ & $\sigma$ \\
\hline 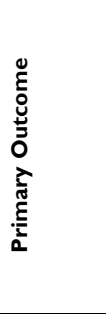 & 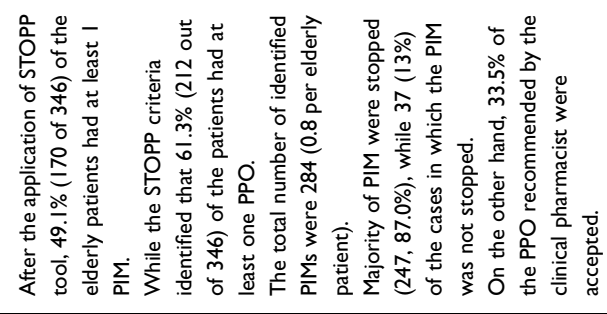 & 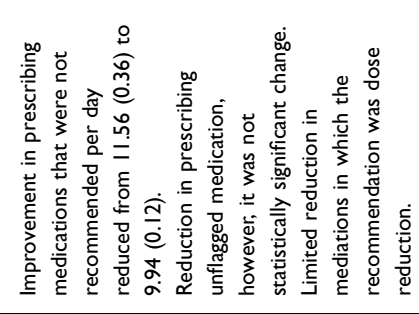 & 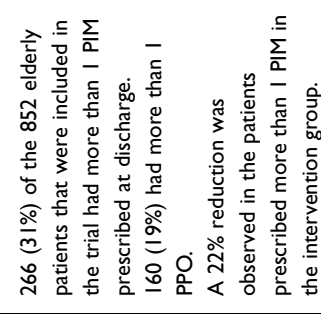 \\
\hline & 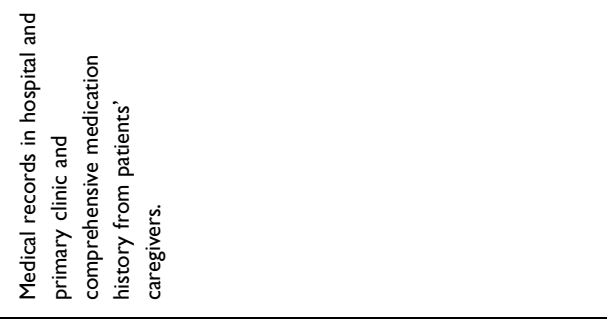 & 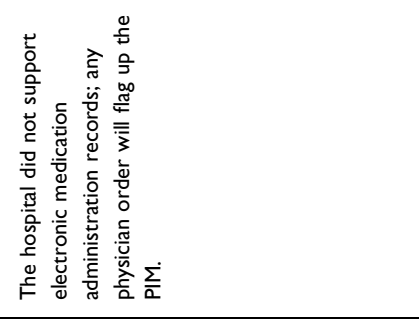 & 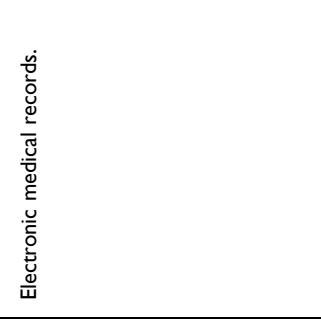 \\
\hline 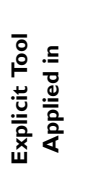 & & 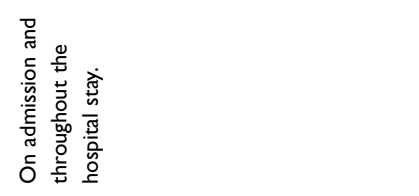 & 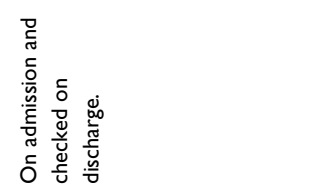 \\
\hline & 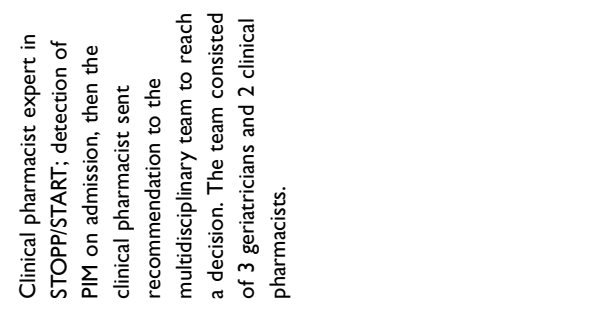 & 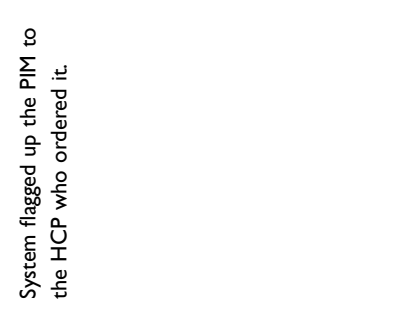 & 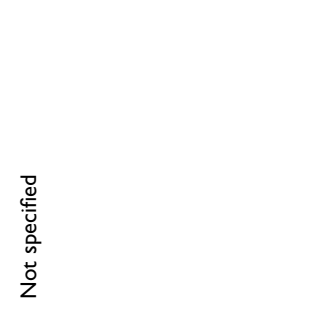 \\
\hline 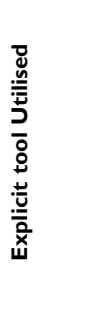 & & 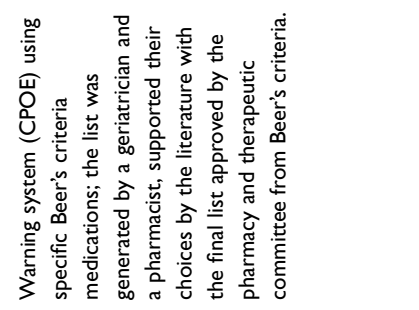 & 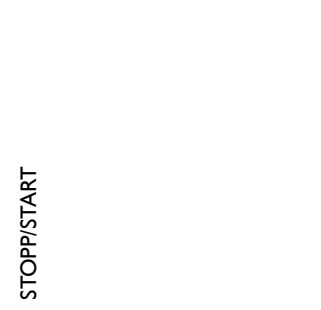 \\
\hline 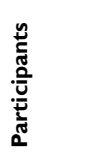 & 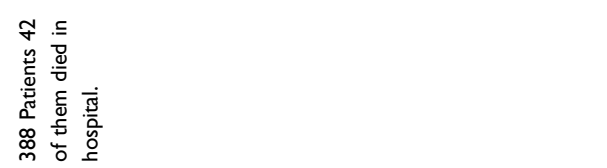 & 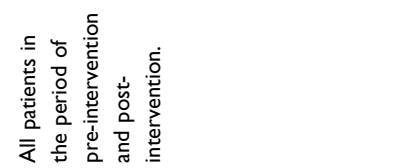 & 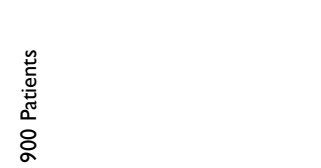 \\
\hline 氞高 & 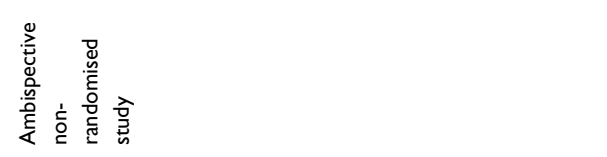 & 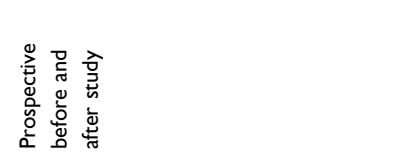 & 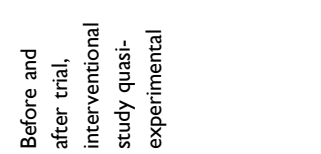 \\
\hline & 惡 & 㐫高 & 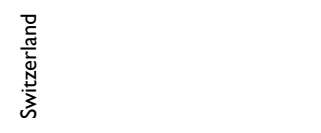 \\
\hline 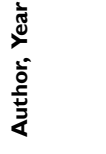 & 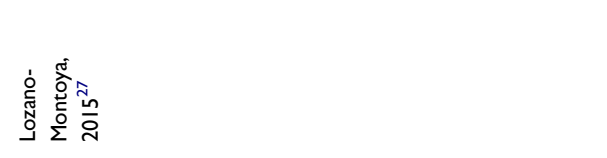 & 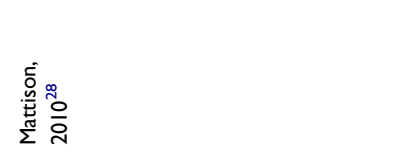 & 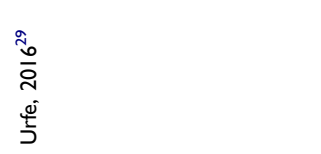 \\
\hline
\end{tabular}




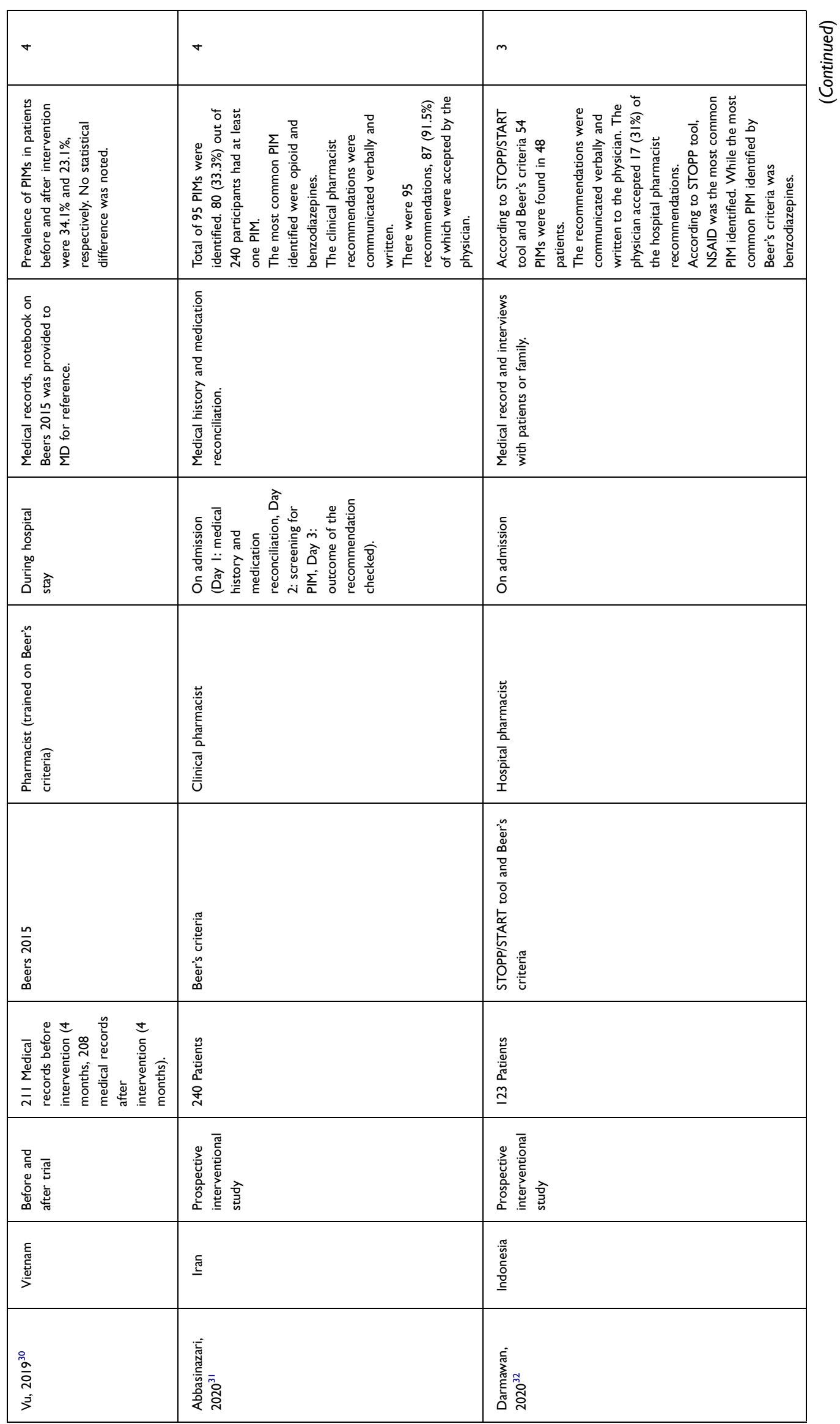




\begin{tabular}{|c|c|c|c|}
\hline 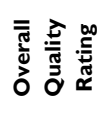 & $\sigma$ & t & r \\
\hline 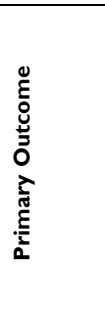 & 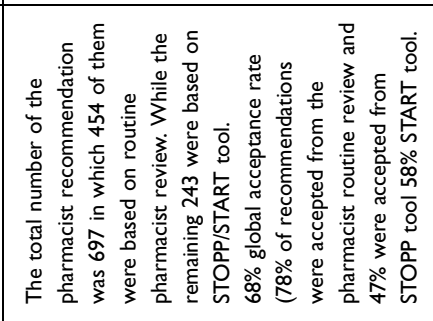 & 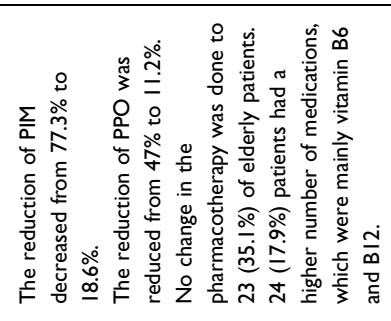 & 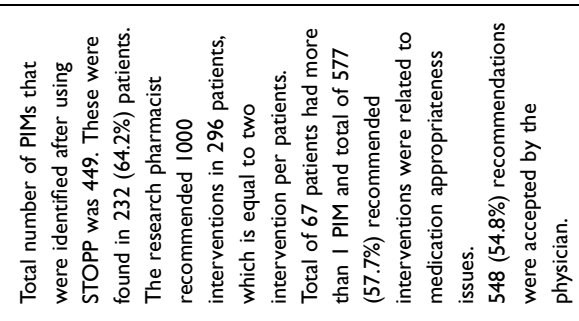 \\
\hline & 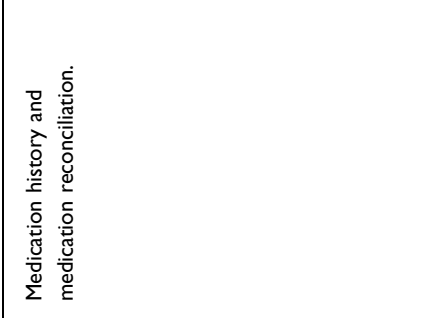 & 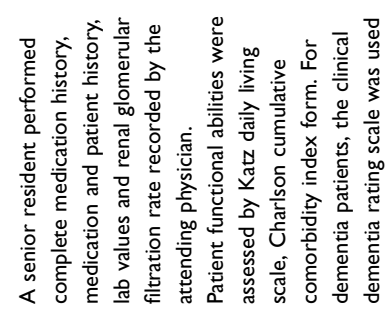 & 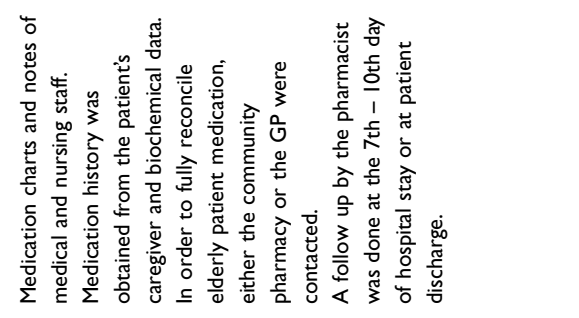 \\
\hline 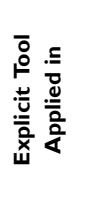 & 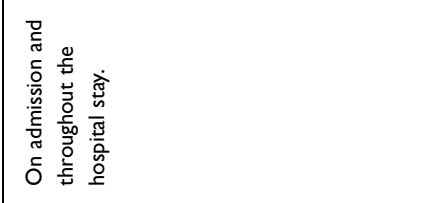 & 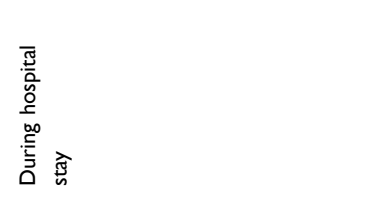 & 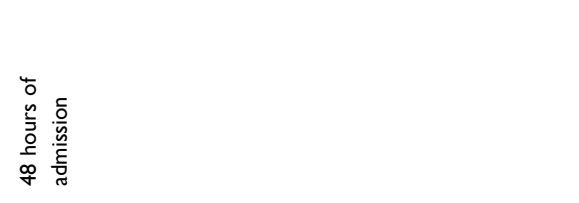 \\
\hline 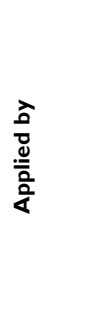 & 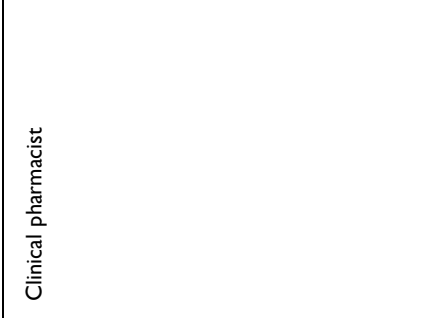 & 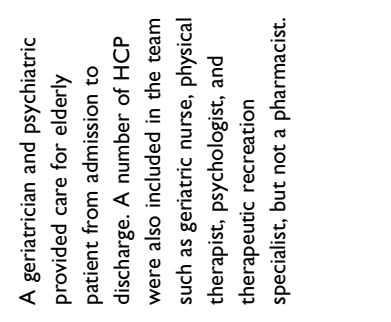 & 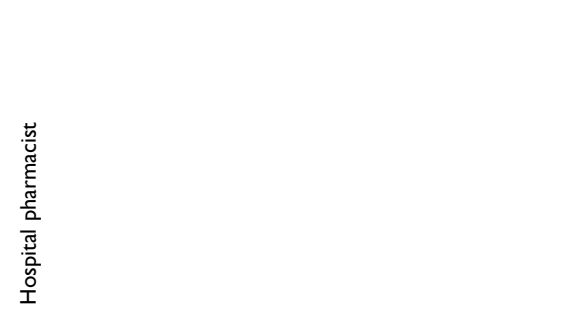 \\
\hline 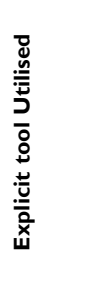 & 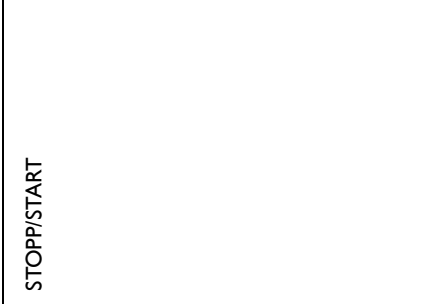 & 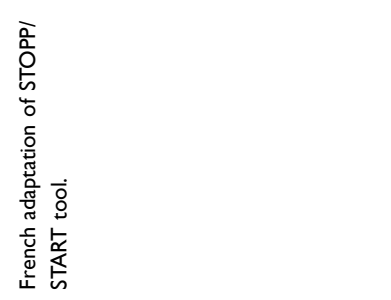 & 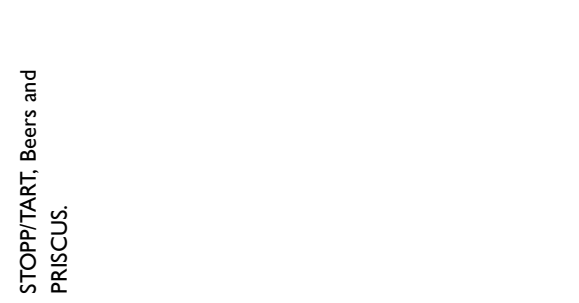 \\
\hline 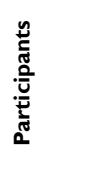 & 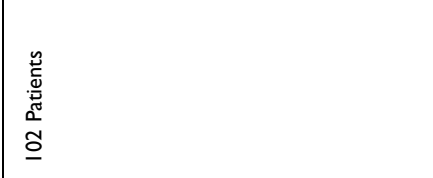 & 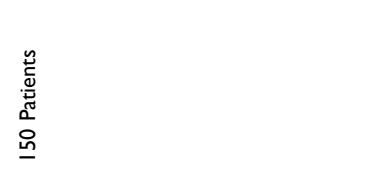 & 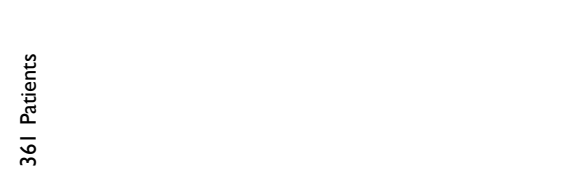 \\
\hline 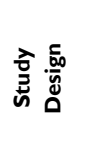 & 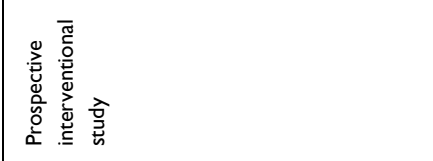 & 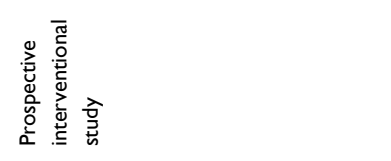 & 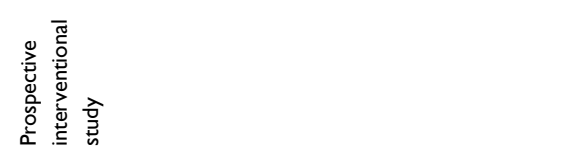 \\
\hline نे & 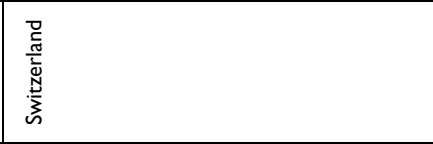 & 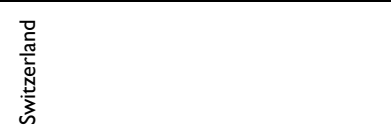 & 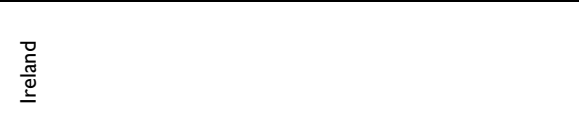 \\
\hline 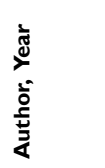 & 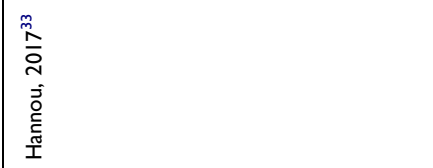 & 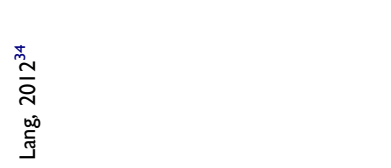 & 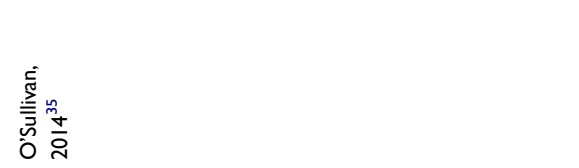 \\
\hline
\end{tabular}




\begin{tabular}{|c|c|c|}
\hline ナ & $m$ & N \\
\hline 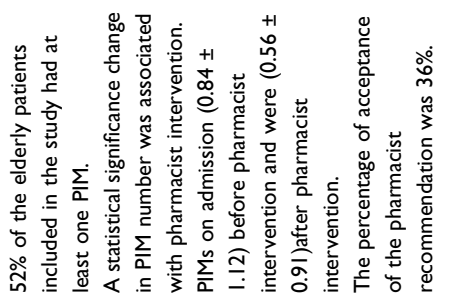 & 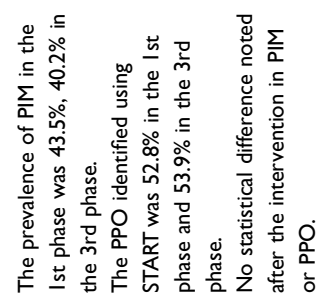 & 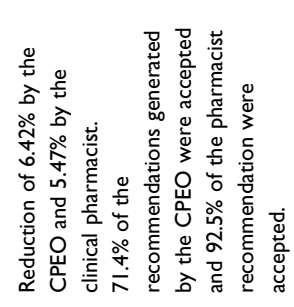 \\
\hline 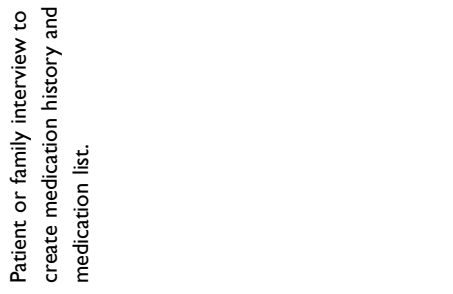 & 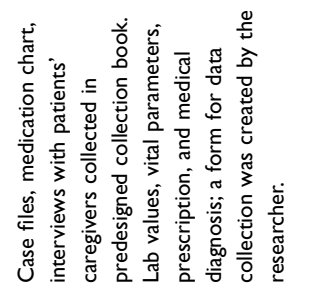 & 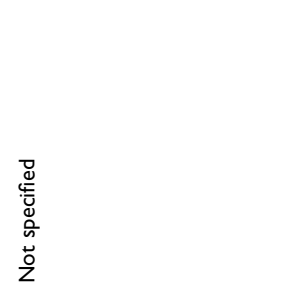 \\
\hline 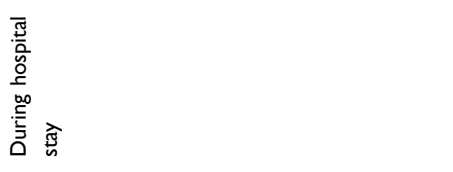 & 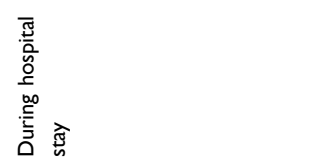 & 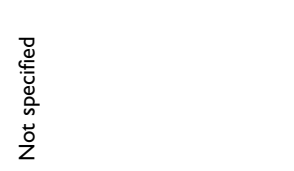 \\
\hline 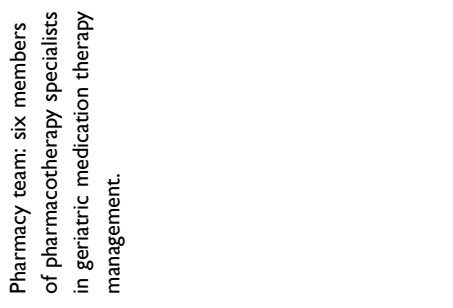 & 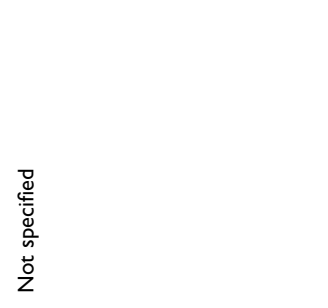 & 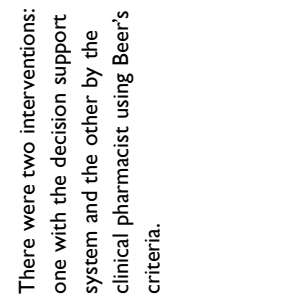 \\
\hline 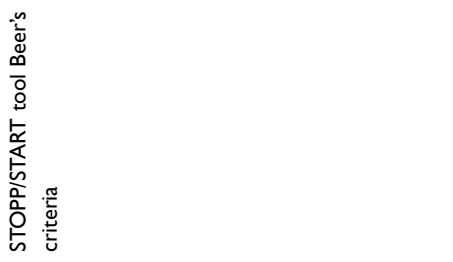 & 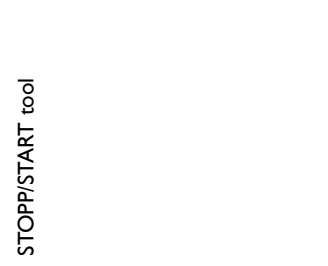 & 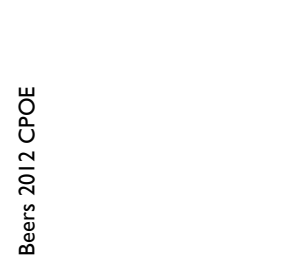 \\
\hline 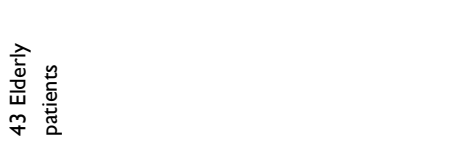 & 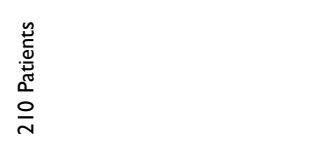 & 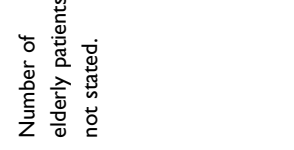 \\
\hline 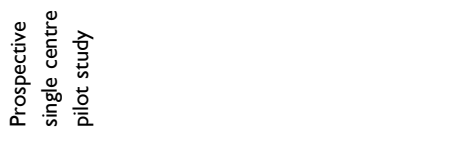 & 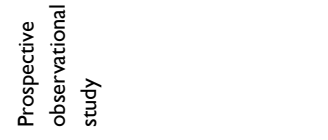 & 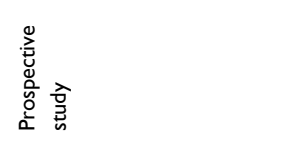 \\
\hline 岁 & $\stackrel{\frac{\pi}{\underline{\underline{n}}}}{\underline{\underline{n}}}$ & 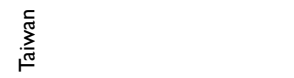 \\
\hline 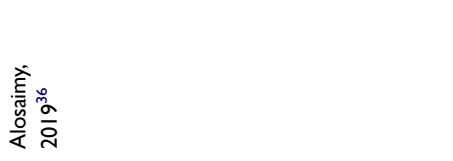 & 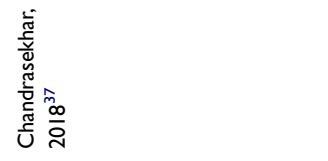 & 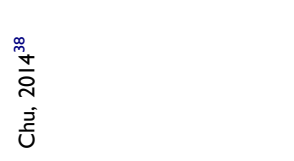 \\
\hline
\end{tabular}




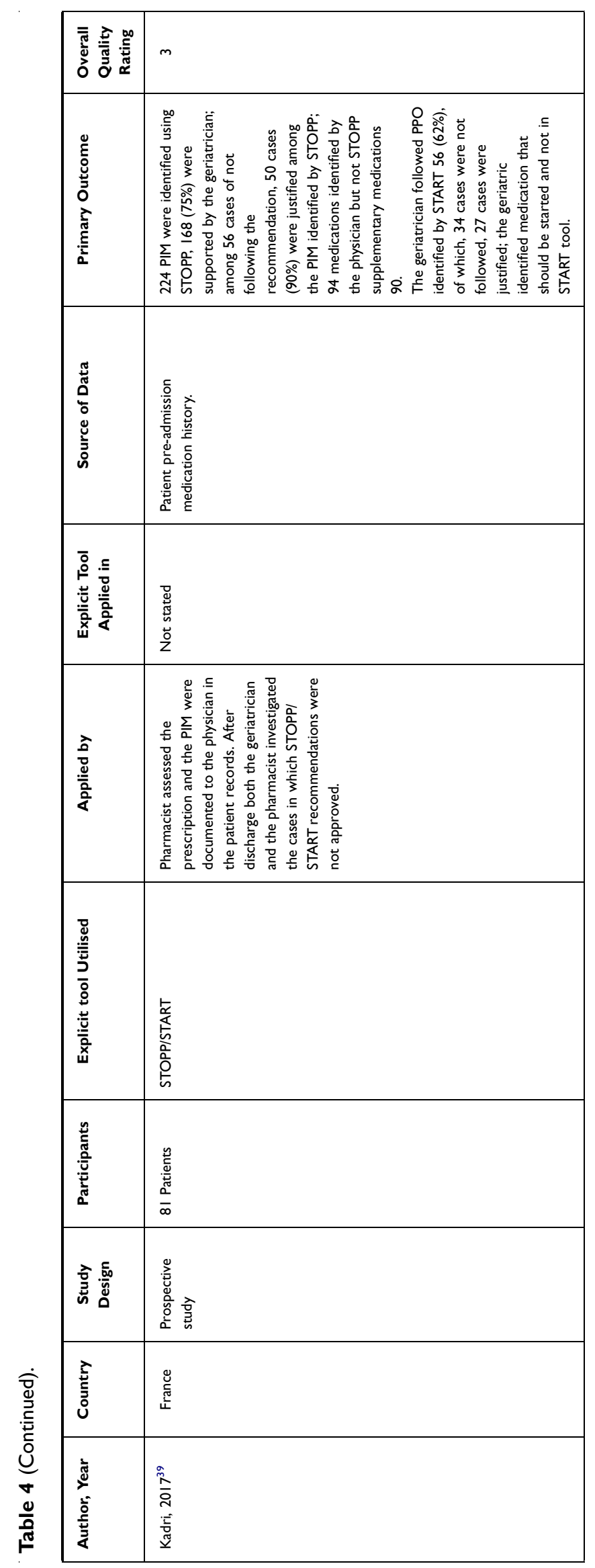




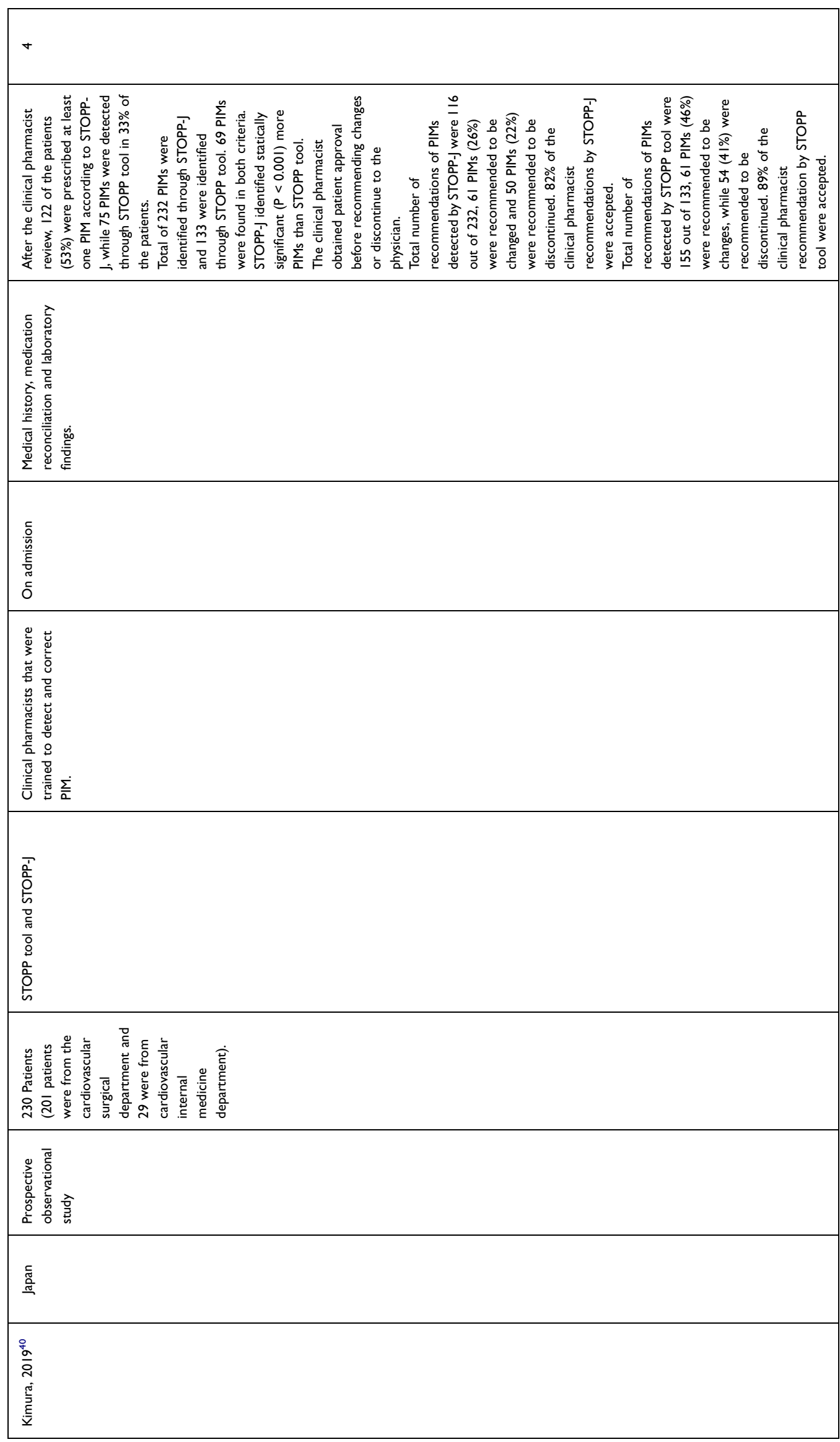




\begin{tabular}{|c|c|c|}
\hline 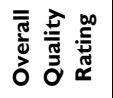 & $\sigma$ & $\sigma$ \\
\hline 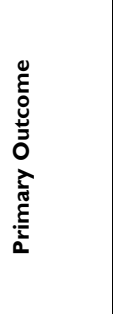 & 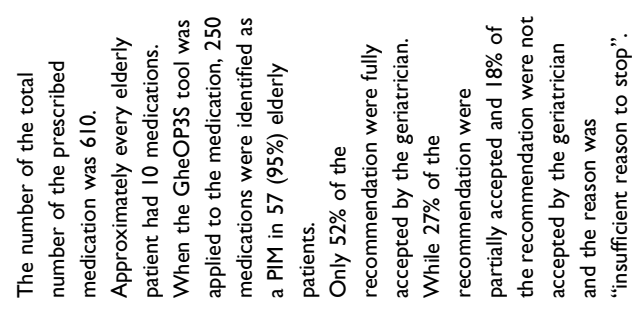 & 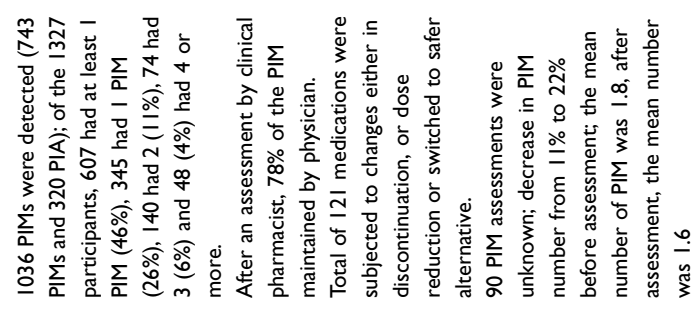 \\
\hline 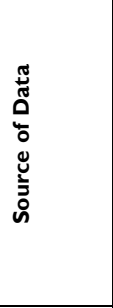 & 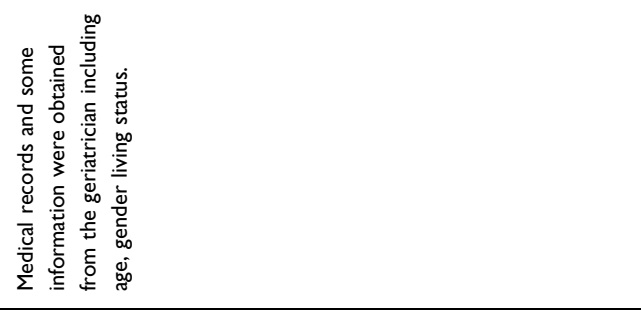 & 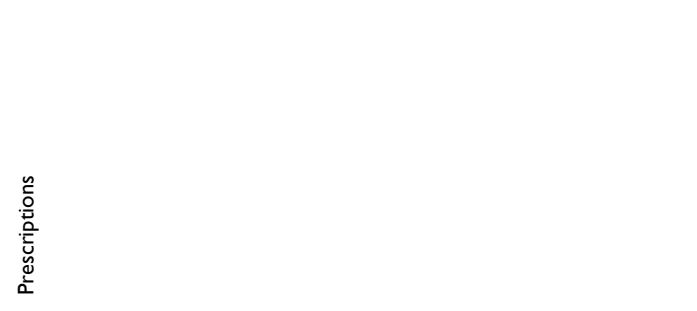 \\
\hline 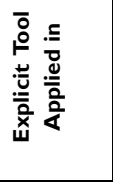 & 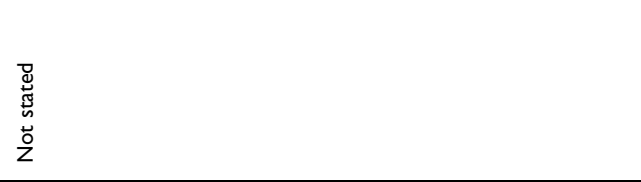 & 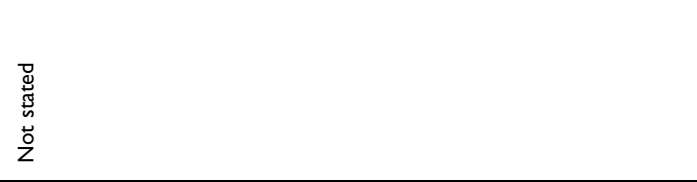 \\
\hline $\begin{array}{l}\widehat{a} \\
\bar{e} \\
\frac{0}{2} \\
\frac{0}{4}\end{array}$ & 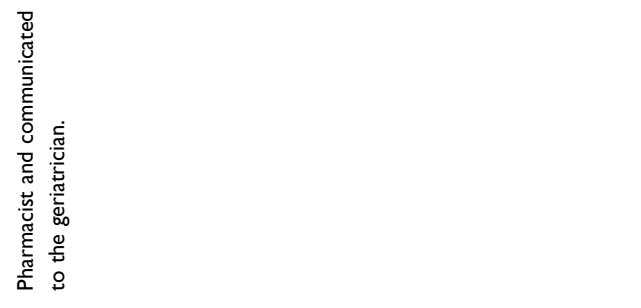 & 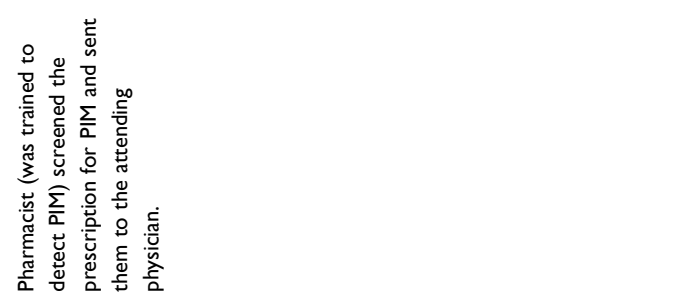 \\
\hline 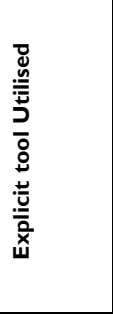 & 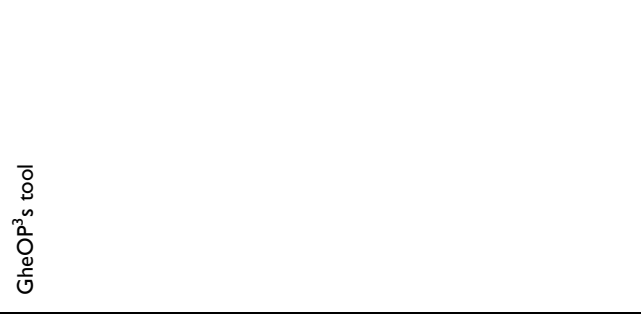 & 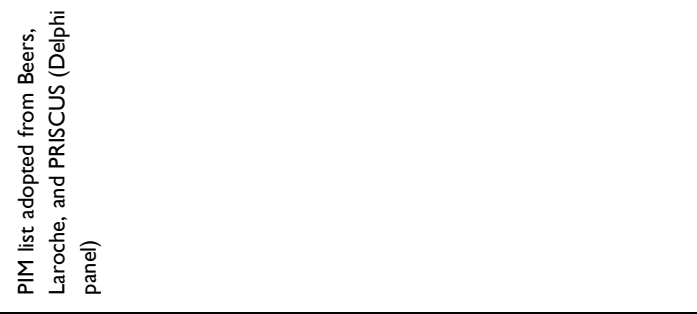 \\
\hline 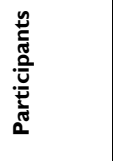 & 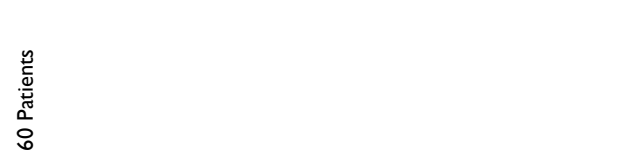 & 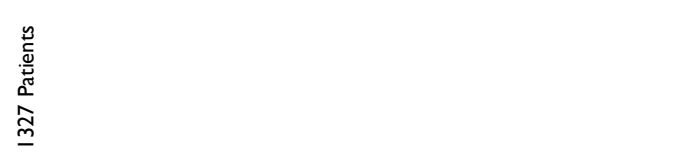 \\
\hline 㝘亳 & 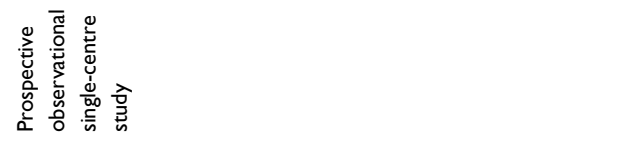 & 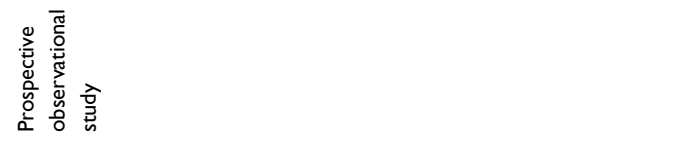 \\
\hline 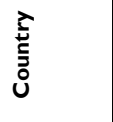 & 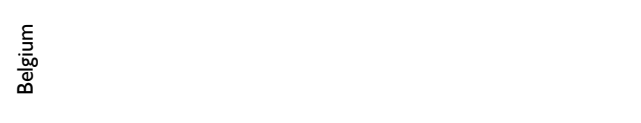 & 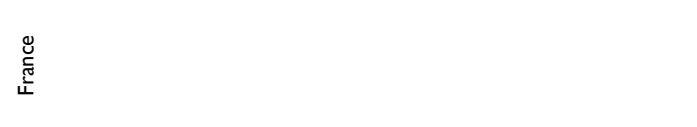 \\
\hline 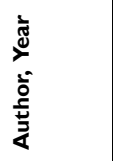 & 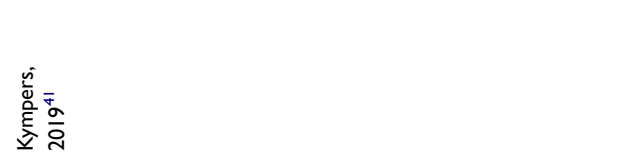 & 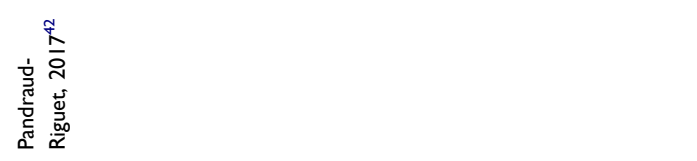 \\
\hline
\end{tabular}




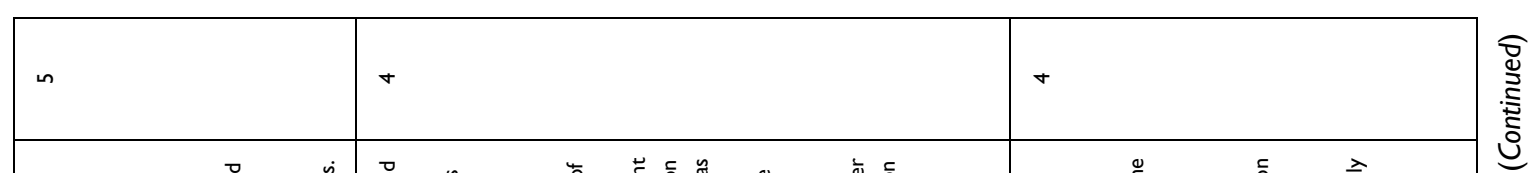

\begin{tabular}{|c|c|c|}
\hline 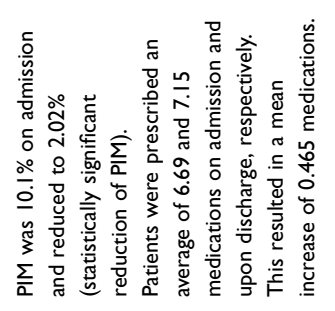 & 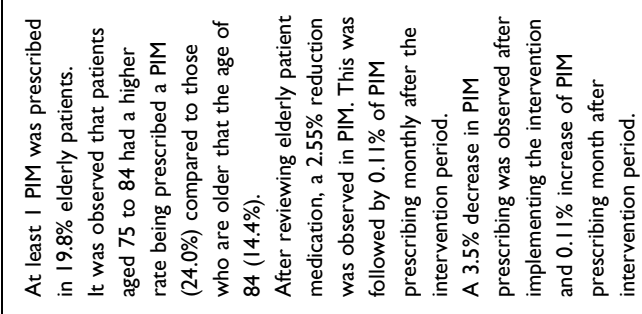 & 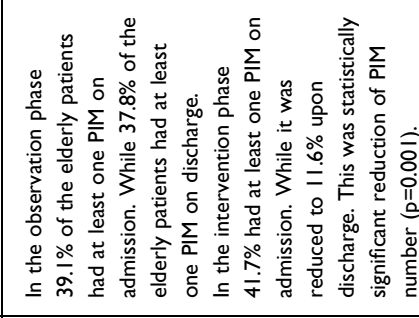 \\
\hline
\end{tabular}

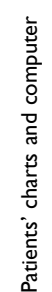

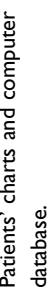

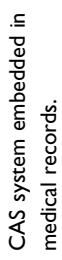

作

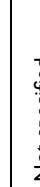

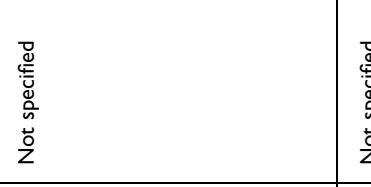

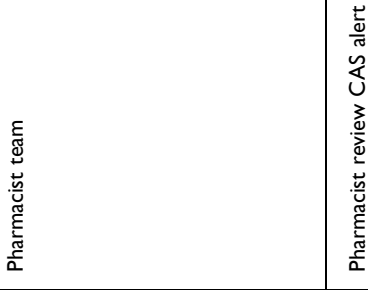

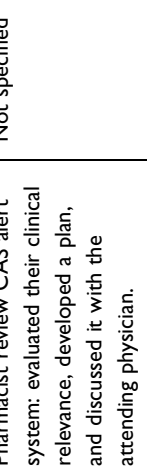

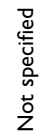

\begin{tabular}{|c|c|}
\hline 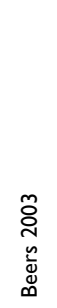 & 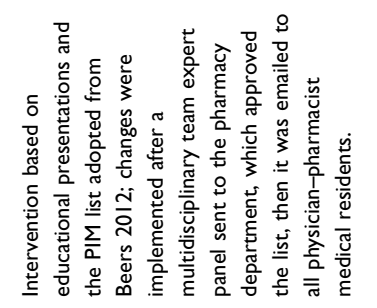 \\
\hline
\end{tabular}

\begin{tabular}{|c|c|c|}
\hline 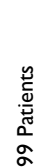 & 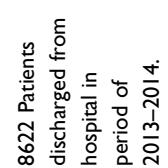 & 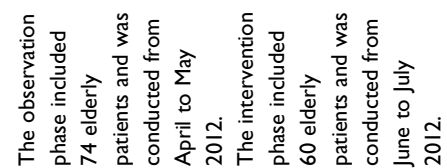 \\
\hline & 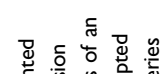 & 莺 \\
\hline
\end{tabular}

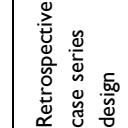

\begin{tabular}{|c|c|c|}
\hline 岵 & 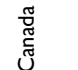 & $\underline{\underline{\mathrm{I}}}$ \\
\hline 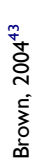 & 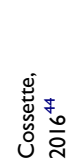 & 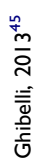 \\
\hline
\end{tabular}




\begin{tabular}{|c|c|}
\hline 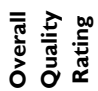 & in \\
\hline 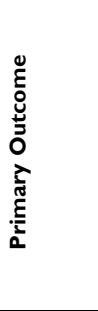 & 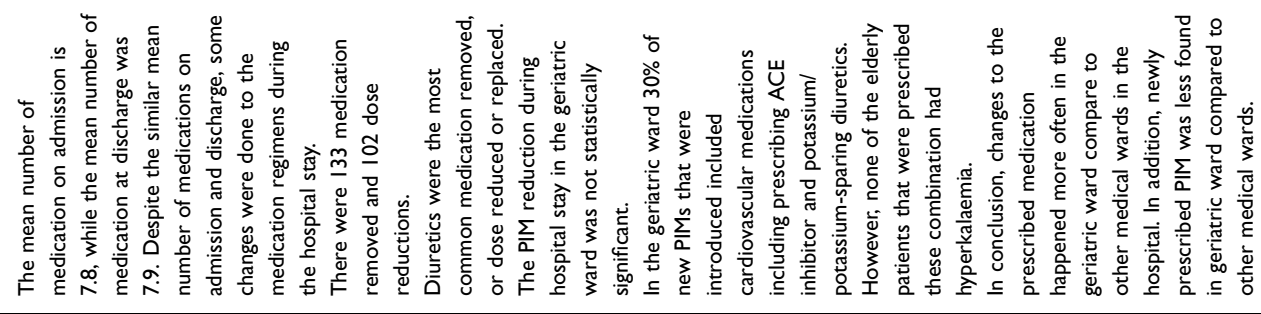 \\
\hline 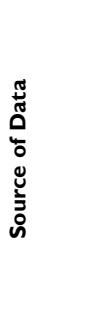 & 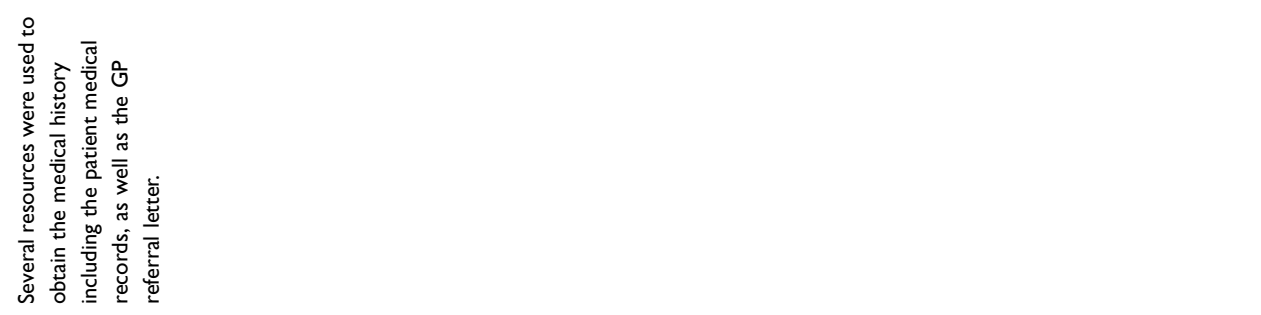 \\
\hline 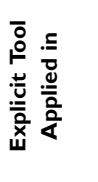 & 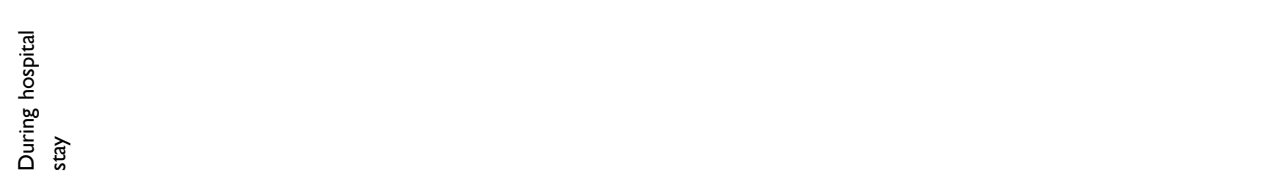 \\
\hline $\begin{array}{l}\text { à } \\
\frac{\overrightarrow{0}}{\bar{a}} \\
\frac{a}{\alpha}\end{array}$ & 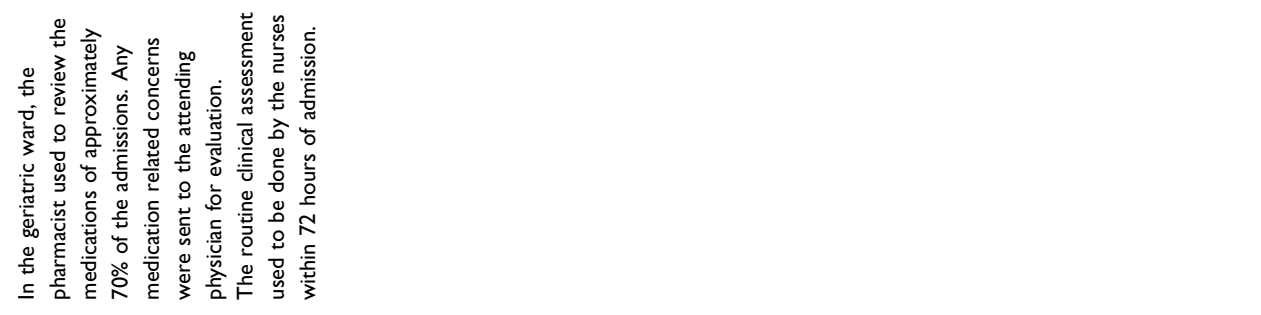 \\
\hline 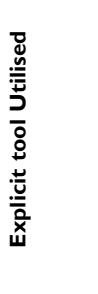 & $\begin{array}{l}\text { U्य } \\
\text { ö } \\
\text { z }\end{array}$ \\
\hline 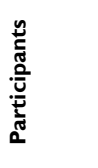 & 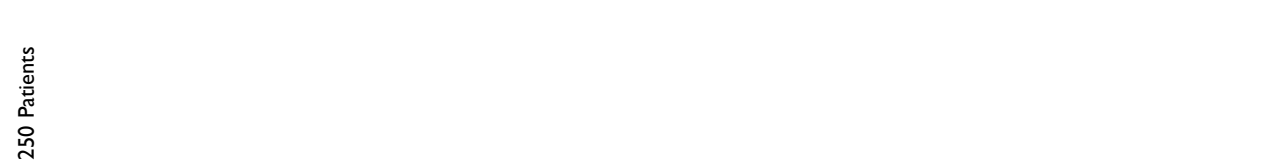 \\
\hline 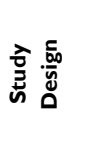 & 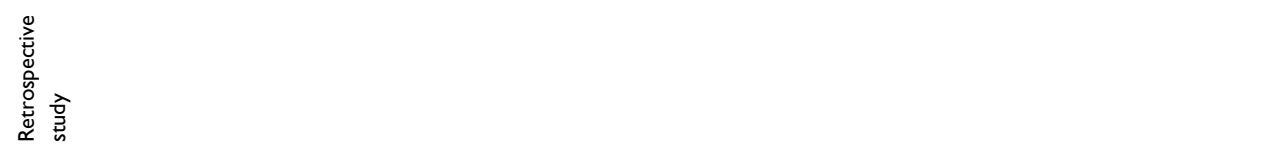 \\
\hline 峷 & $\begin{array}{l}\hat{⿱ 亠 ⿰} \\
\frac{3}{2}\end{array}$ \\
\hline 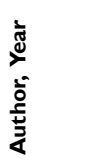 & 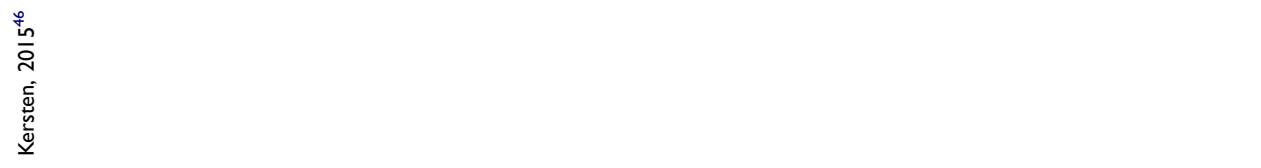 \\
\hline
\end{tabular}




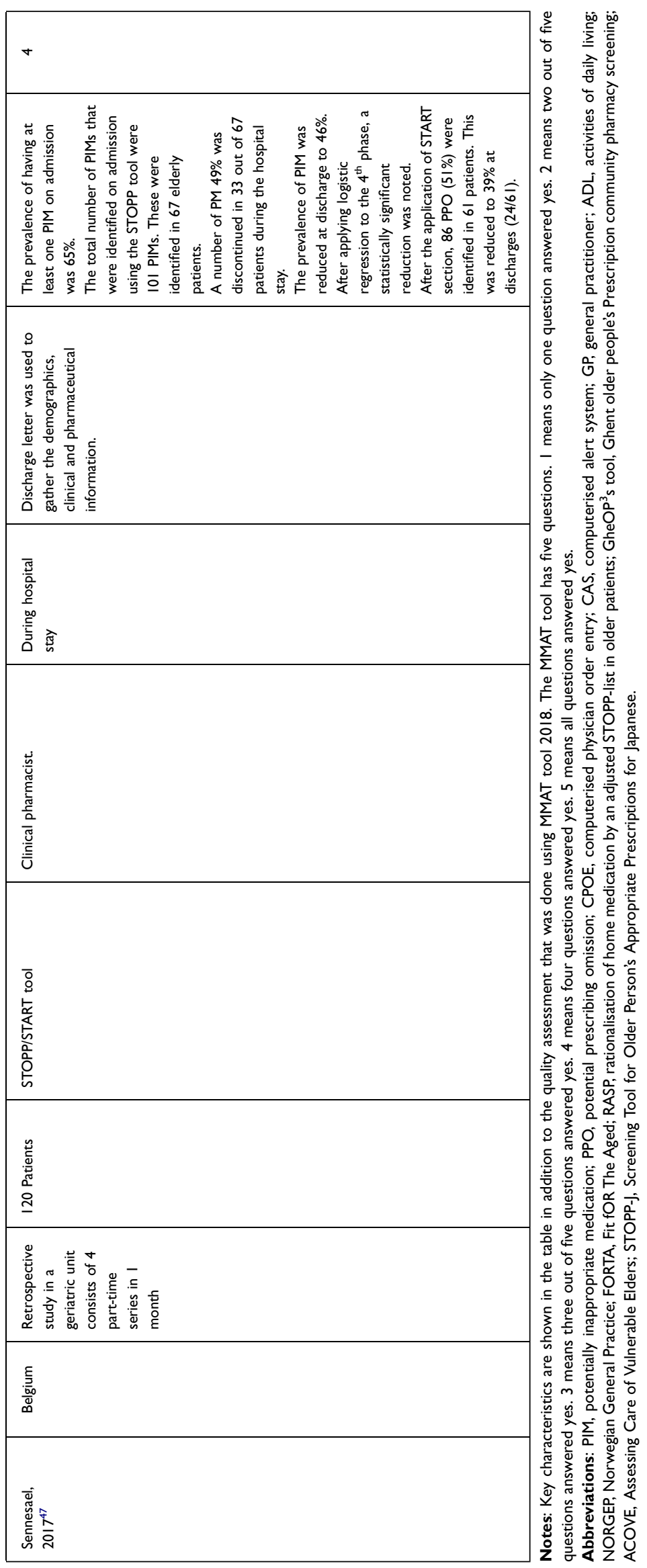


implemented in six studies conducted in the United States, Belgium, Vietnam, Italy, Iran and Taiwan. ${ }^{24,30,31,43,45}$ Two studies used both STOPP/START and Beers criteria. ${ }^{32,36}$ One study implemented the intervention using three different tools STOPP/START, Beers criteria and PRISCUS list, ${ }^{35}$ and two studies used the FORTA list. ${ }^{23,25}$ Other tools were found to be used less frequently in reviewing PIM during hospital stay: the RASP, NORGEP, GheOP3S and STOPP-J tools. The RASP tool was used in a study conducted in Belgium, ${ }^{26}$ NORGEP was used in a study located in Norway, ${ }^{46}$ and the GheOP3S tool, originally designed for community pharmacy screening, was used in a Belgian study. ${ }^{41}$ STOPP-J was developed in Japan and was utilized in a Japanese study. ${ }^{40}$

The most adopted tools were STOPP/START and the Beers criteria. One study originating in Canada adapted STOPP/START and Beers criteria, ${ }^{20}$ two studies that adopted the Beers criteria were conducted in the United States and Canada, ${ }^{28,44}$ one study that adopted STOPP/ START was conducted in Swaziland ${ }^{34}$ and one study conducted in France adopted three tools, PRISCUS, Beers and the Laroche list, through a Delphi panel. ${ }^{42}$ Figure 2 summarizes the tools used in PIM review.

\section{HCP Involved in PIM Review}

HCPs from different specialties were involved in implementing the intervention including physicians, hospital or clinical pharmacists, geriatricians, nurses, physical therapists, psychologist dietitians, occupational therapists, physical therapists, and speech therapists, with hospital or clinical pharmacists conducting the review in most studies (19 out of 27). ${ }^{20,24,26,27,30,33,35,36,38,39,41-44,46,47}$ In three studies, the physician used the STOP/START tool ${ }^{22}$ or the FORTA list. ${ }^{23,25}$ Three studies involved an interdisciplinary team: one included two hospital pharmacists and two geriatricians, ${ }^{42}$ another included nurses, geriatricians, dietitian, occupational therapist, physical therapist, speech therapist and a psychologist, ${ }^{21}$ while the other team consisted of a geriatrician and psychiatrist. ${ }^{34}$ The use of a computerized system instead of HCP to detect PIM was observed in two studies, ${ }^{28,45}$ and two studies did not report the HCP involved in implementing the intervention. ${ }^{29,37}$

\section{PIM Review Process and Stage of Implementation}

The application of explicit tools to reduce PIM during hospitalization was observed on admission (within 48

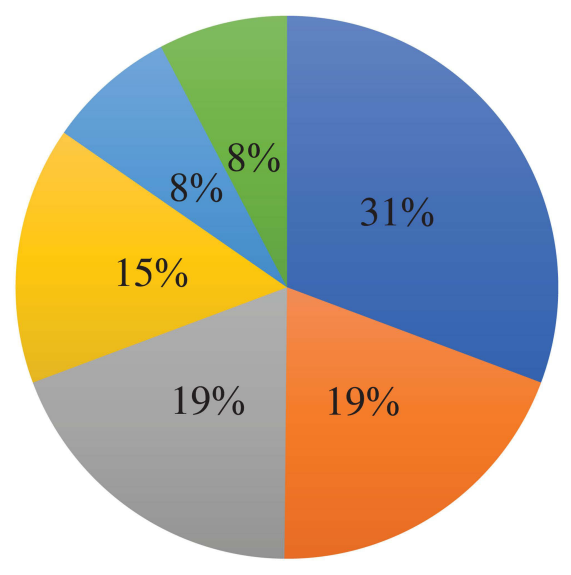

STOPP/START tool Adapted tools

Beers criteria

Others

FORTA list

STOPP/START tool and Beers criteria

Figure 2 Explicit tools that were utilized to review elderly patients' medication in hospitals.

hours of hospitalization) and during the hospital stay, but not on discharge. Ten studies applied explicit tools on admission, ${ }^{21,22,26,28,29,31-33,35,40}$ nine studies during the hospital stay, ${ }^{20,23,24,30,34,36,37,46,47}$ and several studies did not report the stage of hospitalization in which the HCP used the tools. ${ }^{25,38,39,41-45}$

The HCPs involved in PIM detection based their decision on several sources, and any limitations in these sources may influence their clinical decisions. Several studies used only medication history and medication reconciliation ${ }^{26,29-31,33,39,42}$ while other studies depended on medication history and interviews with the patient or caregiver, ${ }^{27,32,36,47}$ and one article added a GP or community pharmacy contact. $^{22}$ One study designed a collection book to record relevant data including medication list, lab values, vital signs and medical diagnosis. ${ }^{37}$ Four studies used clinical examination, medical records and reason for admission in addition to the medication history and interview with patient and caregiver. ${ }^{20,35,41,46}$ Five studies based their decision on patient specific data such as the Mini-Mental State Examination (MMSE), functional dependency, malnutrition, the Katz activities of daily living scale or the Charlson cumulative comorbidity index..$^{21,23-25,34}$ Studies using a computerized system relied mainly on the patient profile in the system..$^{20,28,44,45}$ One study did 


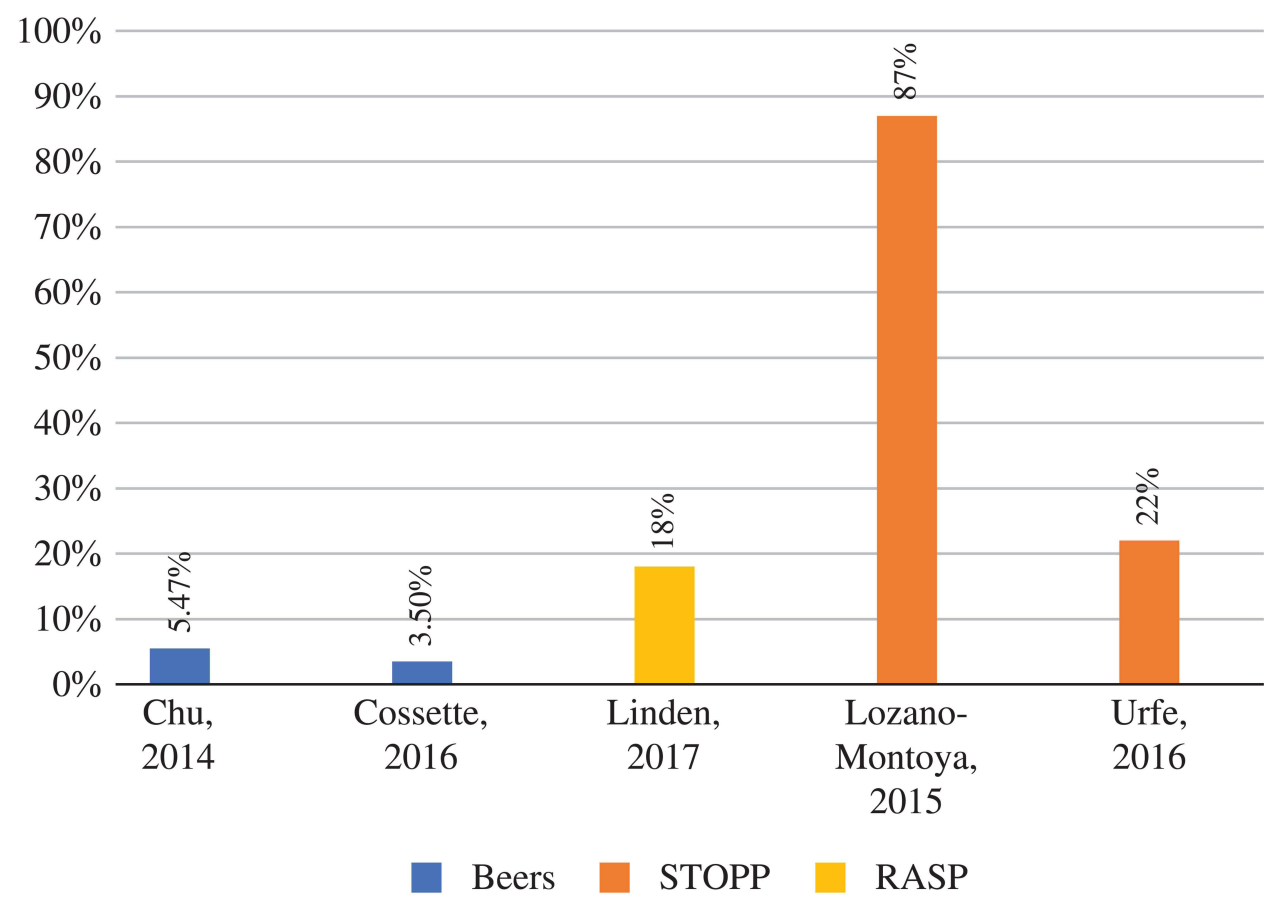

Figure 3 Percentage of PIM reduction in hospitals with the tools used.

not report the type of data used to make their decision of PIM. ${ }^{38}$

The HCP or team included the medication list and other sources in the intervention review and communicated their recommendation verbally or in writing. In eight studies, the PIM was detected using explicit tools and the recommendations were communicated orally to the attending physician,20,21,24,26,33,35,36,44 while in four studies, the PIM was communicated in written form. ${ }^{27,39,41,42}$ Two studies communicated the recommendation verbally and written to the attending physician. ${ }^{31,32}$ Two studies used a computerized system to review PIM and communicated with the physician through an alert system, ${ }^{28,45}$ while in the other two studies, the computerized system and was assessed by the hospital pharmacist to detect PIM and the results communicated verbally to the attending physician. ${ }^{20,44}$ The remaining studies did not specify how the recommendations were communicated to the physician. ${ }^{22,23,25,29,33,34,37,38,40,43,46-48}$

Communicating the PIM review interventions to the GP was observed in two studies, ${ }^{26,34}$ with one study providing a letter to the patient or caregiver. ${ }^{27}$ The other study provided both the GP and the patient or caregiver with PIM changes made during hospitalization. ${ }^{24}$

\section{Intervention Outcomes: PIM Reduction}

Two main methodologies were used to express the outcome of the intervention: the percentage of physician acceptance of intervention and/or the percentage of PIM reduction. Five studies measured the intervention outcome as the percentage reduction in $\mathrm{PIM},{ }^{26,27,29,38,44}$ with the reduction of PIM ranging from $3.5 \%$ up to $87 \%$ (Figures 3 and 4). The study associated with the highest PIM reduction was conducted by the hospital pharmacist, and the intervention was communicated to a team of three geriatricians and two clinical pharmacists. ${ }^{27}$ The lowest PIM reduction was observed in two studies in which the hospital pharmacist detected the PIM and it was sent to the attending physician. ${ }^{38,44}$

Physician acceptance of hospital or clinical pharmacist intervention varied from $36 \%$ to $92.5 \%$, while interventions recommended by the physician generally were more accepted than those of the hospital or clinical pharmacist (91\%) (Figure 5).

Seven studies used the STOPP/START tool and reported the potential prescribing omission (PPO). ${ }^{22,27,33,34,37,39,47}$ PPO were detected in 69 of 382 participants, ${ }^{22} 195$ of 210 patients, $^{37} 90$ of 81 participants, ${ }^{39}$ and 397 of 346 participants. ${ }^{27}$ The highest acceptance rate was for PPO recommendations conducted by a physician at $97 \%$, and the lowest rate of recommendation acceptance was 33.5\% in a study conducted by a clinical pharmacist.

Two studies conducted in the United States and Canada measured the time required to complete the PIM review. One used a computerized system, and the mean time 


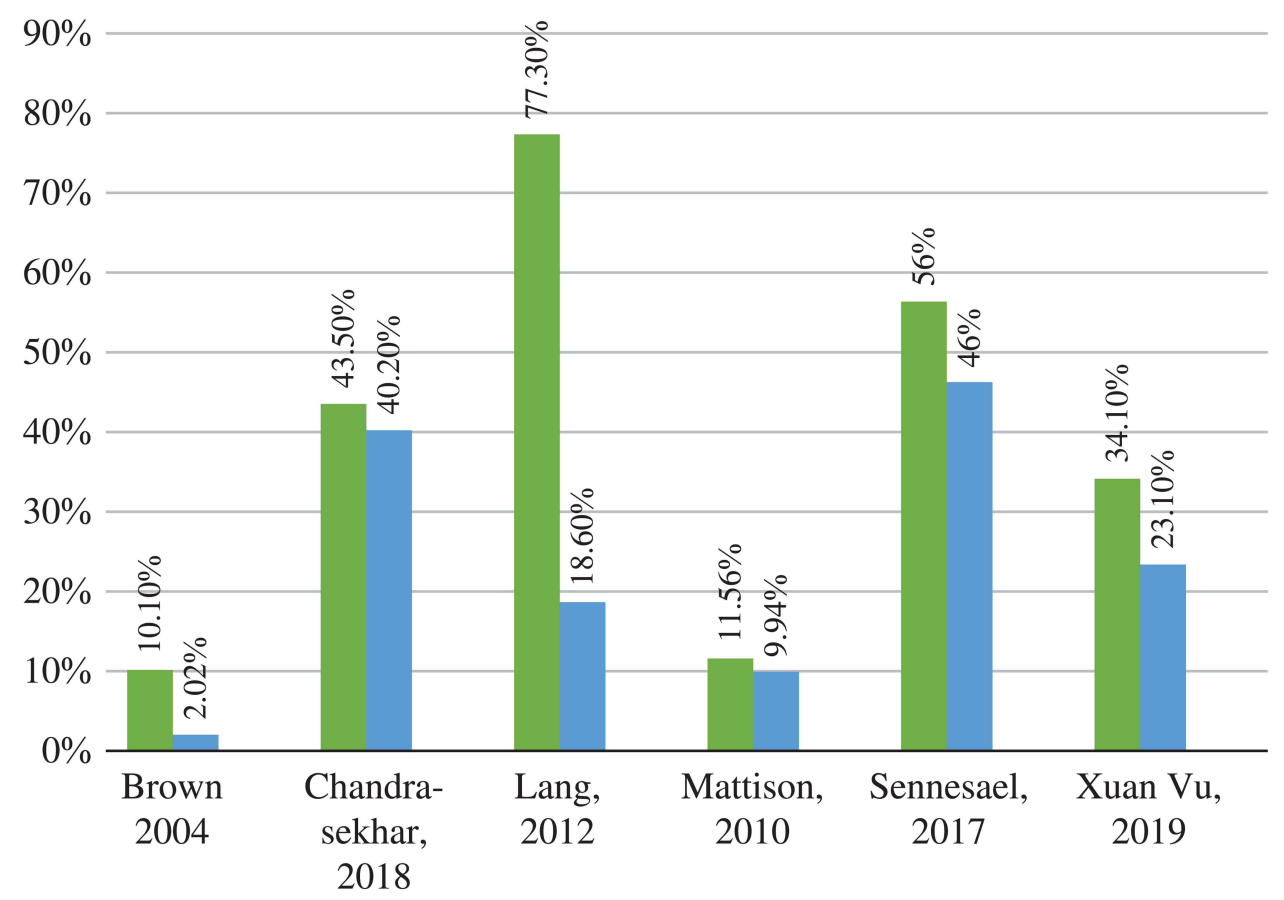

Before intervention After interevntion

Figure 4 Description of PIM reduction before and after tool implementation by HCP in hospitals.

necessary to evaluate PIM was $41 \pm 16$ minutes per patient, ${ }^{20}$ while the other study was a pharmacist-led intervention and needed $49 \pm 16$ minutes per patients to fully complete the PIM review. ${ }^{36}$

\section{Common PIM and Clinical Relevance}

Sixteen articles identified the most commonly detected PIM, 22,26-28,30,31,33,36,37,39-43,45,46 with benzodiazepines being the most common, ${ }^{27,30,31,33,36,41-43,45,46}$ followed by antipsychotics. ${ }^{33,37,41-43}$ Other common PIM included proton pump inhibitors (PPI), ${ }^{2,26}$ digoxin, ${ }^{45}$ NSAID, ${ }^{30,37,40}$ and anticholinergics. ${ }^{28,36,41,46}$

Two studies measured the clinical relevance of the recommendations by tools in the hospitalized older patients. $^{21,41}$ A study evaluated the relevance of the GheOP ${ }^{3} \mathrm{~S}$ tool by two criteria: 1) by the severity of the detected PM, which was evaluated by the treating geriatrician depending on the impact of ADR that could result if the clinical pharmacist did not intervene, and 2) the value of the clinical pharmacist recommendations using a validated patient specific scoring system. ${ }^{41}$ In the first evaluation of clinical relevance, it was shown that $182(73 \%)$ of PIMs were serious and $67(27 \%)$ were classified as significant. $^{41}$ The second evaluation of clinical relevance found six items (2\%) classified as very significant and 235
(94\%) as significant. The second study used the STOPP/ START tool and three experts evaluated the clinical relevance (geriatrician, GP and clinical pharmacist) using a 6point scale system (minor: no benefit or minor benefit; moderate: improvement of the appropriateness of the level of practice or prevention of an adverse drug event of moderate importance; major: prevention of serious morbidity_-including readmission - and serious adverse drug event; extreme: life-saving; deleterious: increased risk of health adverse event; non-applicable). ${ }^{21}$ The experts had access to the patient file to rate the recommendations independently and then discuss the discrepancies. ${ }^{21}$ The expert panel classified as major: 29\%, moderate: 37 , minor: $5 \%$, deleterious $8 \%{ }^{21}$

\section{Clinical and Non-Clinical Outcomes}

Out of 25 studies included in this review, 8 examined the clinical effect of PIM reduction. ${ }^{20,22,27,28,30,33-35}$ Only one study looked at non-clinical effect, cost, of PIM reduction. $^{16}$

\section{Clinical Outcomes} PIM Reduction and Activities of Daily Living (ADL) The ADL was measured by three articles, ${ }^{23,25,27}$ with one study reporting that non-statistically significant differences 


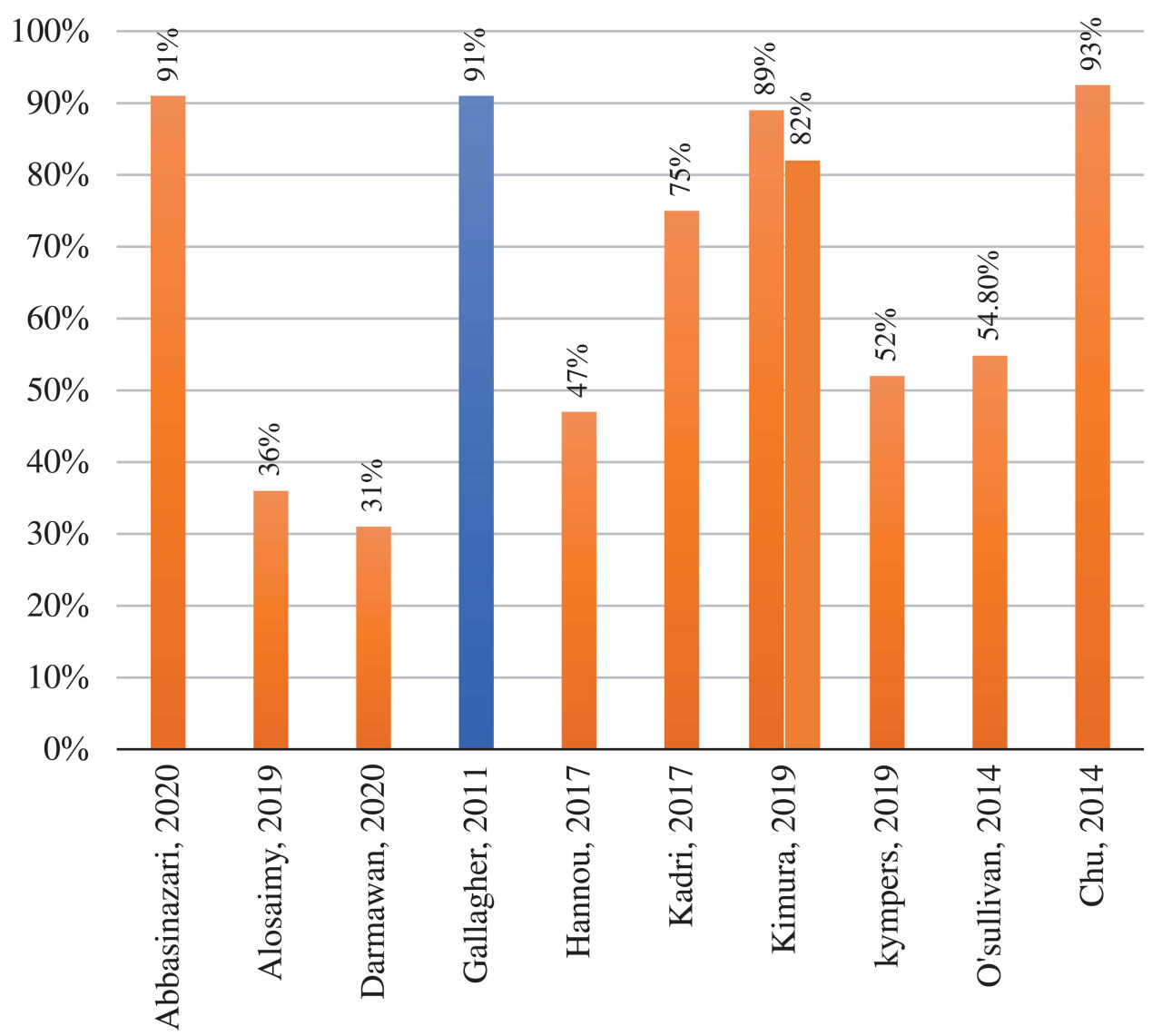

By Physician By Pharmacist

Figure 5 The percentage accepting the recommendation for PIM reduction.

were found between ADL in the intervention and control groups (Barthel Index 70 in control and 80 in intervention group $\mathrm{P}<0.220){ }^{23}$ Two studies found a statistically significant relation between PIM reduction and improved ADL. ${ }^{25,27}$

\section{PIM Reduction and Falls}

The effect of reducing PIM in falls in older hospitalised patients was documented in three studies. ${ }^{22,23,26}$ Falls were lower in the intervention group; however, none of the three studies reported a statistically significant change in falls after the PIM reduction.

\section{Hospitalization and Readmission}

A number of studies measured the impact on hospital stay, ${ }^{20,22}$ in-hospital mortality, ${ }^{20,26,29}$ ED visits, ${ }^{20,27,33}$ and readmissions ${ }^{20,24,26,29}$ as well as GP visits. ${ }^{22}$ Two studies found a non-statistically significant link between PIM reduction and hospital stay. ${ }^{20,22}$ Similarly, a non-statistically significant link between PIM reduction and in-hospital mortality was reported in three studies. ${ }^{20,26,29}$ Four studies assessed the impact of PIM reduction on hospital readmission, and all four reported no statistical difference in hospital readmission between the intervention and control groups. ${ }^{20,24,26,29}$ Similarly, studies evaluating the association of ED visits and PIM reduction reported no difference or minimal difference, not statistically significant, between older patients in the intervention and those who were in the control groups. ${ }^{20,24,26}$ One study assessed GP visits and their relation to PIM reduction and reported that patients in the intervention group had fewer GP visits compared to patients in the control group; however, it was not statistically significant $(\mathrm{P}=0.063){ }^{22}$

\section{Non-Clinical Outcomes}

Assessing the cost associated with each intervention was rarely addressed in the literature, with one study measuring the total cost saving resulting from reducing PIM in terms of healthcare resources utilisation and medication. ${ }^{36}$ The study reported that the cost saving from the clinical intervention yielded approximately $£ 63,000-144,000$ as well as $£ 68,000$ annual medication savings. ${ }^{36}$ 


\section{Discussion}

This systematic review presented data from 25 studies that examined PIM review using explicit tools in hospitalized patients over 65 years old. This review specifically explored the explicit tools used in reviewing older patient medication in hospitals, which is usually done on admission and during the hospital stay. The hospital or clinical pharmacist was often found to review the medication of older patients. Variable PIM reduction percentages were reported by studies in this review.

In this review, several tools were identified as being used to detect PIM, with the STOPP/START tool being the most common. One reason for the preference of the STOPP/START tool over other tools might be the availability of a START list, which includes medications that should be prescribed for older adults. Moreover, STOPP/ START tools have been shown to have higher PIM detection and higher clinical relevance than Beers criteria. ${ }^{48}$ However, another comparison study examined the detectability of PIM comparing two tools, STOPP/START and PIM-check, revealing that PIM-check detected three times more PIM than STOPP/START. ${ }^{43}$ This could be due to the fact that the PIM-check tool was developed by an international panel of experts, which may add another dimension to the detectability of PIM in research done in a country other the one where the tool was developed. A number of explicit tools were designed be used in a specific healthcare setting, but they are still effective in reducing PIM in other than the intended settings. For example, the Beers criteria were specifically designed to be used in nursing homes, but when it was used in hospitals, a statistically significant reduction in PIM was noted, ${ }^{38}$ even when it was integrated with a computerized system. ${ }^{45}$ In addition, the GheOP ${ }^{3} \mathrm{~S}$ tool was proposed to be used in community pharmacies, but when it was applied in a hospital setting, it resulted in the physician accepting $52 \%$ of the recommendations, comparable to the acceptance rate of the STOPP/ START tool. ${ }^{33}$ Nonetheless, careful adoption of explicit tools needs to be considered in healthcare settings other than those for which the tool was designed. ${ }^{24}$ Only two studies in this review have utilized the computerised automatic PIM detection. This could highly aid the HCP in PIM detection in hospitalized elderly. Future studies need to consider investigating the outcomes of the application of such automatic PIM detection in daily practice.

Another finding of this review is the point at which the PIM review occurred during hospitalization: on admission or during hospital stay. Several studies did not disclose when the PIM review took place, highlighting the lack of information regarding the most appropriate time to review PIM during hospitalization. It is difficult to recommend when the right time to detect PIM would take place because each point of the hospital journey has its pros and cons. For example, a full medication history is performed on admission, allowing identification of the causative agent that precipitated the admission. ${ }^{49,50}$ However, conducting the review during the hospital stay could help in improving the prescribing practice; many studies proved that the PIM increases during hospital stay. ${ }^{51,52}$ Among the various healthcare settings, hospital stay can be identified as the best place where PIM can be reviewed, ${ }^{49}$ because if there is a need to stop a PIM, the patient can be monitored by the HCP. The inpatient environment is considered one of the facilitators for deprescribing in hospitals, as the availability of resources and the patient mentioning to ensure safe deprescribing. ${ }^{53}$ Additionally, geriatricians and hospital pharmacist acknowledge their role to be more proactive and responsible in avoiding harm to patients. A study was done in a hospital in the UK to investigate whether medication reviews in a hospital lead to deprescribing of medications related to increase the risk of falls. ${ }^{54}$ The study recruited 100 patients over the age of 70 who were admitted due to falls, and these patients were followed prospectively. Medication reconciliations were done by pharmacists for $80 \%$ of the patients, and medication reviews were done for $86 \%$ of the patients. There are a number of patients $(2 \%)$ that the doctor documented "review" to the pharmacist, but it was not carried out. Sixty-five out of 100 elderly patients were on medications that increased the risk of falls. After a comprehensive medication review, fall-risk medications of 23 of the 65 patients were reduced. After applying an analysis of the data, the medication review by the pharmacist was found statistically significant in reducing the fall-risk medications $(\mathrm{P}=0.002) .{ }^{54}$ Deprescribing in 65 years and over inpatients shows promising outcomes as concluded Grazarin and colleagues study that was aimed at evaluating inpatient deprescribing initiatives. ${ }^{55}$ These studies emphasise how the hospital could be an opportunity to reduce PIM through collaborative work between physicians and pharmacists. On the other hand, a qualitative study reported that some physicians in primary care express some concerns regarding potentially adverse outcomes and follow-up from deprescribing and fast pace in daily practice. ${ }^{56}$ 
This review identified that physicians, pharmacist, and multidisciplinary teams are involved in the PIM review process in hospitals. Similarly to what was obtained in Thomas and Thomas (2019) review and Santos and colleagues (2019) review. ${ }^{11,57}$ To optimize the care of hospitalized elderly, HCPs need geriatric pharmacotherapy programs and training. ${ }^{58,59}$ Another important consideration is treating the patient in a holistic manner, as some physicians and specialist tend to focus their efforts on managing the acute state and reason for hospitalization. ${ }^{58,60,61}$

The reduction of PIM varied between studies, ranging from $3.5 \%$ up to $85 \%$, and the recommendation acceptance ranged from $36 \%$ up to $93 \%$. It is interesting to note that the physician has a higher PIM reduction and recommendation acceptance than the pharmacist, which may indicate a lack of effective communication. ${ }^{62}$ Additionally, the power dynamics might influence the relationship between pharmacists and physicians, ${ }^{63}$ as some physicians lack knowledge about the professional role and job description of the pharmacist. A possible way to strengthen the pharmacist-physician relationship is through a simulation involving face-to-face pharmacist and physician interaction, ${ }^{64}$ which could also increase the pharmacists' confidence, helping them to be more proactive in collaboration with the physician. Physicians prefer face-to-face communication in terms of providing recommendations, as evident from a semi-structured interview:

The pharmacist comes and writes a note for you, but it's not done face to face, and it actually is a bit antagonistic if anything .... having post-it [notes] stuck on things saying please review this, please review that, we all hate notes, everyone hates it, so I think that could be done better. So more pharmacy input, but more integrated pharmacy input. $^{59}$

The method in which the recommendation is communicated to the physician is either written or verbal and could affect whether or not the recommendation status is approved.

Several explanations could elucidate the variation in PIM reduction. Firstly, the suitability of the explicit tool in the practice or setting. Advanced health care systems may reflect less PIM reduction as their practice is optimised by the guidelines and policies. Similarly, with geriatric hospitals or geriatric wards. The availability of the alternatives medications to PIM could lead to higher reductions. One of the influential aspect in the management and reduction of PIM is the patient choice as some of them might be physiologically attached to the prescribed medication. Fear and concerns are considered barriers to the reducing of the elderly's medication as some of the elderly fear that they might miss the benefits of the deprescribed medication in the future. ${ }^{65-67}$ Another aspect that might hinder the deprescribing is related to patient expectations, as they are unfamiliar with the process of reducing instead of adding medication. ${ }^{65-68}$ One of the studies pointed out the influences on willingness to deprescribe, which could be carers or friends that have unsuccessfully stopped their medication. ${ }^{64}$ This will negatively influence the amount of medication stopped by physicians, which may reflect the variation in PIM reduction in this review.

Effective communication between primary care clinics and hospitals is essential for continuous healthcare. ${ }^{69}$ In several studies, the GP reported lack of sufficient information in discharge letters. ${ }^{70,71}$ Providing a letter to the patient and the GP indicating the changes and reasons behind stopping PIM is important so as not to reinitiate what was already discontinued. Engaging and empowering the patient will also help to sustain the changes, since many patients are reluctant to change or stop their medications. ${ }^{59}$ Additionally, improving the communication between hospitals and GPs is essential to increase patient safety. ${ }^{50,72}$ This can be improved by using a form that includes all vital information that needs to be sent to the GP.

The present review confirmed that only a few studies highlighted the clinical outcomes of PIM reduction. It was noted that reducing PIM was associated with improved ADL, fewer falls, fewer readmissions, and fewer GP visits. These findings were similar to what was reported by Hill-Taylor et al (2013) in a review that aimed to examine the impact of the STOPP/START tool application. ${ }^{73}$ The limited number of studies measuring PIM reduction clinical outcomes could be due to the nature of outcomes, which is often hard to evaluate. Additionally, it requires considerable effort of observation and reporting which could be labour and time consuming. Moreover, loss of follow-up could be one of the limitations investigating the clinical outcomes of PIM reduction. Despite that, it is vital to assess those outcomes to support deprescribing PIMs. Future studies need to address the clinical outcomes of such interventions.

It is worth noting that some of the study findings were not statistically significant. Confidence intervals and $\mathrm{P}$ values in the studies helped in assessing the clinical significance of the study results ${ }^{74}$; however, these statistical tools aid the decision but do not make the decision. Some effects are not statistically significant, but clinically, they 
can make a meaningful difference to the patient's health. ${ }^{75}$ For a careful clinical decision and to deeply understand the impact of PIM reduction, more studies are required to investigate both the short- and long-term effects of reducing PIM as well as the economic aspect of this intervention.

\section{Strengths and Limitations}

This systematic review explored the application of different explicit tools in hospitals to review PIM, narratively synthesising the data to allow the identification of key aspects of the application of the explicit tools in the hospital, such as whether the HCP was involved in PIM review and what tools were utilized, the stage of hospitalization in which the PIM review occurred, as well as the clinical relevance of the PIM detected. In addition, numerous gaps and areas for future studies were noted. There were some limitations at the methodological level that are common in this kind of review, since non-English articles were excluded. The studies included in this review were located mostly in Europe and the United States, where the healthcare systems are more developed compared to other countries. In terms of analysis, studies used different methods to express the outcomes of the PIM review, so it was not possible to pool the data and perform statistical analysis for a meta-analysis.

\section{Conclusion}

PIM is a serious healthcare issue for older patients and can be improved through various means such as the use of implicit or explicit tools. This systematic review explored the practice of reviewing PIM in hospitalized patients using explicit tools, which showed promising outcomes in terms of improving PIM. Future studies need to consider the application of explicit tools in other healthcare settings setting to confirm the findings. PIM reduction is linked to better overall health of older patients and has a positive influence in reducing falls. Nonetheless, more studies need to be conducted to further investigate the outcomes of reviewing PIM at different levels, as well as assessing the clinical and cost-effectiveness of using such tools to minimize PIM.

\section{Data Sharing Statement}

Supplementary file upon request from the corresponding author (data extraction in Microsoft excel format).

\section{Disclosure}

The authors declare that they have no competing interests.

\section{References}

1. Jensen LD, Andersen O, Hallin M, Petersen J. Potentially inappropriate medication related to weakness in older acute medical patients. Int J Clin Pharm. 2014;36(3):570-580. doi:10.1007/s11096-014-9940-y

2. Renom-Guiteras A, Thürmann PA, Miralles R, et al. Potentially inappropriate medication among people with dementia in eight European countries. Age Ageing. 2018;47(1):68-74. doi:10.1093/ageing/afx147

3. Morgan SG, Hunt J, Rioux J, Proulx J, Weymann D, Tannenbaum C. Frequency and cost of potentially inappropriate prescribing for older adults: a cross-sectional study. CMAJ Open. 2016;4(2):E346-E351. doi:10.9778/cmajo.20150131

4. Harrison SL, Kouladjian O'Donnell L, Milte R, et al. Costs of potentially inappropriate medication use in residential aged care facilities. BMC Geriatr. 2018;18(1):9. doi:10.1186/s12877-0180704-8

5. Fick DM, Cooper JW, Wade WE, Waller JL, Maclean JR, Beers MH. Updating the Beers criteria for potentially inappropriate medication use in older adults: results of a US consensus panel of experts. Arch Intern Med. 2003;163(22):2716-2724. doi:10.1001/ archinte.163.22.2716

6. Piau A, Huet Y, Gallini A, Andre L, Vellas B, Nourhashemi F. Optimization of drug therapy in elderly individuals admitted to a geriatric unit. Clin Interv Aging. 2017;12:1691-1696. doi:10.2147/CIA.S132309

7. Goodwin MA, Stange KC, Zyzanski SJ, Crabtree BF, Borawski EA, Flocke SA. The Hawthorne effect in direct observation research with physicians and patients. J Eval Clin Pract. 2017;23(6):1322-1328. doi:10.1111/jep.12781

8. Laroche ML, Charmes JP, Nouaille Y, Fourrier A, Merle L. Impact of hospitalisation in an acute medical geriatric unit on potentially inappropriate medication use. Drugs Aging. 2006;23(1):49-59. doi:10.2165/00002512-200623010-00005

9. Onatade R, Auyeung V, Scutt G, Fernando J. Potentially inappropriate prescribing in patients on admission and discharge from an older peoples' unit of an acute UK hospital. Drugs Aging. 2013;30(9):729737. doi:10.1007/s40266-013-0097-5

10. Komagamine J. Prevalence of potentially inappropriate medications at admission and discharge among hospitalised elderly patients with acute medical illness at a single centre in Japan: a retrospective crosssectional study. BMJ Open. 2018;8(7):e021152. doi:10.1136/bmjopen-2017-021152

11. Dos Santos NS, Marengo LL, Moraes FDS, Barberato-Filho S. Interventions to reduce the prescription of inappropriate medicines in older patients. Rev Saúde Pública. 2019;53:7.

12. Sallevelt BTGM, Huibers CJA, Knol W, van Puijenbroek E, Egberts T, Wilting I. Evaluation of clarity of the STOPP/START criteria for clinical applicability in prescribing for older people: a quality appraisal study. BMJ Open. 2020;10(2):e033721. doi:10.1136/bmjopen2019-033721

13. By the 2019 American Geriatrics Society Beers Criteria ${ }^{\circledR}$ Update Expert Panel. American geriatrics society 2019 updated AGS Beers criteria ${ }^{\circledR}$ for potentially inappropriate medication use in older adults. J Am Geriatr Soc. 2019;67(4):674-694. doi:10.1111/jgs.15767

14. O'Mahony D, O'Sullivan D, Byrne S, O'Connor MN, Ryan C, Gallagher P. STOPP/START criteria for potentially inappropriate prescribing in older people: version 2. Age Ageing. 2015;44(2):213218. doi:10.1093/ageing/afu145

15. Motter FR, Fritzen JS, Hilmer SN, Paniz ÉV, Paniz VMV. Potentially inappropriate medication in the elderly: a systematic review of validated explicit criteria. Eur J Clin Pharmacol. 2018;74(6):679-700. doi:10.1007/s00228-018-2446-0

16. Higgins J, Green S, editors. Cochrane Handbook for Systematic Reviews of Interventions. England: Wiley-Blackwell; 2008.

17. Hong QN, Pluye P, Fàbregues S, et al. Mixed methods appraisal tool (MMAT), version 2018. Canada: IC Canadian Intellectual Property Office; 2018. 
18. Hong QN, Fàbregues S, Bartlett G, et al. The mixed methods appraisal tool (MMAT) version 2018 for information professionals and researchers. Educ Inf. 2018;34(4):285-291. doi:10.3233/EFI-180221

19. Page MJ, McKenzie JE, Bossuyt PM, Boutron I, Hoffmann TC, Mulrow CD, et al. The PRISMA 2020 statement: an updated guideline for reporting systematic reviews. BMJ. 2017 Mar 29;372-n71.

20. Cossette B, Éthier J-F, Joly-Mischlich T, et al. Reduction in targeted potentially inappropriate medication use in elderly inpatients: a pragmatic randomized controlled trial. Eur J Clin Pharmacol. 2017;73 (10):1237-1245. doi:10.1007/s00228-017-2293-4

21. Dalleur O, Boland B, Losseau C, et al. Reduction of potentially inappropriate medications using the STOPP criteria in frail older inpatients: a randomised controlled study. Drugs Aging. 2014;31 (4):291-298. doi:10.1007/s40266-014-0157-5

22. Gallagher PF, O'Connor MN, O’Mahony D. Prevention of potentially inappropriate prescribing for elderly patients: a randomized controlled trial using STOPP/START criteria. Clin Pharmacol Ther. 2011;89(6):845-854. doi:10.1038/clpt.2011.44

23. Michalek C, Wehling M, Schlitzer J, Frohnhofen H. Effects of 'Fit fOR The Aged' (FORTA) on pharmacotherapy and clinical endpoints-a pilot randomized controlled study. Eur J Clin Pharmacol. 2014;70(10):1261-1267. doi:10.1007/s00228-014-1731-9

24. Spinewine A, Swine C, Dhillon S, et al. Effect of a collaborative approach on the quality of prescribing for geriatric inpatients: a randomized, controlled trial. J Am Geriatr Soc. 2007;55(5):658-665.

25. Wehling M, Burkhardt H, Kuhn-Thiel A, et al. VALFORTA: a randomised trial to validate the FORTA (Fit fOR The Aged) classification. Age Ageing. 2016;45(2):262-267. doi:10.1093/ageing/afv200

26. der Linden LV, Decoutere L, Walgraeve K, et al. Combined use of the rationalization of home medication by an adjusted STOPP in older patients (RASP) list and a pharmacist-led medication review in very old inpatients: impact on quality of prescribing and clinical outcome. Drugs Aging. 2016;2(34):123-133.

27. Lozano-Montoya I, Vélez-Diaz-Pallarés M, Delgado-Silveira E, Montero-Errasquin B, Cruz Jentoft AJ. Potentially inappropriate prescribing detected by STOPP-START criteria: are they really inappropriate? Age Ageing. 2015;44(5):861-866. doi:10.1093/ageing/afv079

28. Mattison MLP, Afonso KA, Ngo LH, Mukamal KJ. Preventing potentially inappropriate medication use in hospitalized older patients with a computerized provider order entry warning system. Arch Intern Med. 2010;170(15):1331-1336. doi:10.1001/archinternmed.2010.244

29. Urfer M, Elzi L, Dell-Kuster S, Bassetti S. Intervention to improve appropriate prescribing and reduce polypharmacy in elderly patients admitted to an internal medicine unit. PLoS One. 2016;11(11): e0166359. doi:10.1371/journal.pone.0166359

30. Xuan Vu T, Bui Thi Huong Q. The effect of the pharmacist's intervention on potentially inappropriate medication use in older adults using the 2015 Beers criteria. Pharm Sci Asia. 2019;46(1):54-61. doi:10.29090/psa.2019.01.017.0023

31. Abbasinazari M, Tavana A. Identification and acceptance of suggested therapeutic alternatives of potentially inappropriate medications amongst hospitalised geriatric patients in Iran. J Pharm Pract Res. 2020;50(1):75-77. doi:10.1002/jppr.1583

32. Darmawan E, Ahmad H, Perwitasari DA, Kusumawardani N. Pharmacist intervention can reduce the potential use of inappropriate drugs medications in Indonesian geriatric patients. J App Pharm Sci. 2020;10(1):88-95.

33. Hannou S, Voirol P, Pannatier A, et al. Pharmacist intervention acceptance for the reduction of potentially inappropriate drug prescribing in acute psychiatry. Int J Clin Pharm. 2017;39(6):12281236. doi:10.1007/s11096-017-0513-8

34. Lang PO, Vogt-Ferrier N, Hasso Y, et al. Interdisciplinary geriatric and psychiatric care reduces potentially inappropriate prescribing in the hospital: interventional study in 150 acutely ill elderly patients with mental and somatic comorbid conditions. J Am Med Dir Assoc. 2012;13(4):406.e1-406.e7. doi:10.1016/j.jamda.2011.03.008
35. O'Sullivan D, O'Mahony D, O'Connor MN, et al. The impact of a structured pharmacist intervention on the appropriateness of prescribing in older hospitalized patients. Drugs Aging. 2014;31(6):471-481. doi:10.1007/s40266-014-0172-6

36. Alosaimy S, Vaidya A, Day K, Stern G. Effect of a pharmacist-driven medication management intervention among older adults in an inpatient setting. Drugs Aging. 2019;36(4):371-378. doi:10.1007/s40266018-00634-9

37. Chandrasekhar D, Samjas M, Pattani D. Evaluation of potentially inappropriate medications among hospitalized geriatric patients in tertiary care referral hospital using STOPP/START criteria. Clin Epidemiol Glob Health. 2018. Available from http://www.sciencedirect.com/ science/article/pii/S2213398418300216. Accessed October 15, 2021.

38. Chu LL, Su HC, Wang HY. Construct a CPOE decision supporting and monitoring system to decrease PIMS used in hospitalized elderly patients. Value Health. 2014;17(7):A754-A755. doi:10.1016/j. jval.2014.08.220

39. Kadri B, Tritz T, Lecoeur A, Mercier FL, Cudennec T. CP-030 assessment of medical care for older patients in an acute geriatric department with the stopp/start criteria. Eur J Hosp Pharm. 2017;24 (Suppl 1):A13.

40. Kimura T, Ogura F, Kukita Y, et al. Efficacy of pharmacists' assessment and intervention based on Screening Tool for Older Persons' Appropriate Prescriptions for Japanese compared with Screening Tool of Older Persons' potentially inappropriate prescriptions criteria version 2 in older patients with cardiovascular disease. Geriatr Gerontol Int. 2019;19(11):1101-1107.

41. Kympers C, Tommelein E, Van Leeuwen E, Boussery K, Petrovic M, Somers A. Detection of potentially inappropriate prescribing in older patients with the GheOP ${ }^{3} \mathrm{~S}$-tool: completeness and clinical relevance. Acta Clin Belg. 2019;74:126-136.

42. Pandraud-Riguet I, Bonnet-Zamponi D, Bourcier E, et al. Monitoring of potentially inappropriate prescriptions in older inpatients: a French multicenter study. J Am Geriatr Soc. 2017;65(12):2713-2719. doi:10.1111/jgs. 15081

43. Brown B, Earnhart J. Pharmacists and their effectiveness in ensuring the appropriateness of the chronic medication regimens of geriatric inpatients [Internet]. 2004 [cited April 2, 2019]. Available from: https://www.ingentaconnect.com/content/ascp/tcp/2004/00000019/ 00000005/art00003. Accessed October 15, 2021.

44. Cossette B, Bergeron J, Ricard G, et al. Knowledge translation strategy to reduce the use of potentially inappropriate medications in hospitalized elderly adults. J Am Geriatr Soc. 2016;64(12):24872494. doi:10.1111/jgs.14322

45. Ghibelli S, Marengoni A, Djade CD, et al. Prevention of inappropriate prescribing in hospitalized older patients using a computerized prescription support system (INTERcheck $\left({ }^{\circledR}\right)$ ). Drugs Aging. 2013;30 (10):821-828. doi:10.1007/s40266-013-0109-5

46. Kersten H, Hvidsten LT, Gløersen G, Wyller TB, Wang-Hansen MS. Clinical impact of potentially inappropriate medications during hospitalization of acutely ill older patients with multimorbidity. Scand J Prim Health Care. 2015;33(4):243-251. doi:10.3109/ 02813432.2015 .1084766

47. Sennesael A-L, Dalleur O, Henrard S, Artoisenet C, Schoevaerdts D, Spinewine A. Implementing a screening tool to improve prescribing in hospitalized older patients: a pilot study. Int J Clin Pharm. 2018;40 (1):15-19. doi:10.1007/s11096-017-0563-y

48. Boland B, Guignard B, Dalleur O, Lang PO. Application of STOPP/ START and Beers criteria: compared analysis on identification and relevance of potentially inappropriate prescriptions. 2016 [cited April 2, 2019]; Available from: https://www.sciencedirect.com/science/arti cle/pii/S1878764916300286. Accessed October 15, 2021.

49. Henschel F, Redaelli M, Siegel M, Stock S. Correlation of incident potentially inappropriate medication prescriptions and hospitalization: an analysis based on the PRISCUS list. Drugs Real World Outcomes. 2015;2(3):249-259. doi:10.1007/s40801-015-0035-4 
50. Ní Chróinín D, Neto HM, Xiao D, et al. Potentially inappropriate medications (PIMs) in older hospital in-patients: prevalence, contribution to hospital admission and documentation of rationale for continuation. Australas J Ageing. 2016;35(4):262-265. doi:10.1111/ ajag. 12312

51. Pérez T, Moriarty F, Wallace E, McDowell R, Redmond P, Fahey T. Prevalence of potentially inappropriate prescribing in older people in primary care and its association with hospital admission: longitudinal study. BMJ. 2018;363:k4524. doi:10.1136/bmj.k4524

52. Rothberg MB, Pekow PS, Liu F, et al. Potentially inappropriate medication use in hospitalized elders. J Hosp Med. 2008; Mar (2):91-102. doi:10.1002/jhm.290

53. Scott S, Twigg MJ, Clark A, et al. Development of a hospital deprescribing implementation framework: a focus group study with geriatricians and pharmacists. Age Ageing. 2020;49(1):102-110. doi:10.1093/ageing/afz133

54. Marvin V, Ward E, Poots AJ, Heard K, Rajagopalan A, Jubraj B. Deprescribing medicines in the acute setting to reduce the risk of falls. Eur J Hosp Pharm. 2017;24(1):10-15. doi:10.1136/ejhpharm2016-001003

55. Gazarin M, Devin B, Tse D, et al. Evaluating an inpatient deprescribing initiative at a rural community hospital in Ontario. Can Pharm J. 2020;153(4):224-231. doi:10.1177/1715163520929734

56. Wallis KA, Andrews A, Henderson M. Swimming against the tide: primary care physicians' views on deprescribing in everyday practice. Ann Fam Med. 2017;15(4):341-346. doi:10.1370/afm.2094

57. Thomas RE, Thomas BC. A systematic review of studies of the STOPP/START 2015 and American geriatric society Beers 2015 criteria in patients $\geq 65$ years. Curr Aging Sci. 2019;12(2):121-154. doi:10.2174/1874609812666190516093742

58. Al Shemeili S, Klein S, Strath A, Fares S, Stewart D. An exploration of health professionals' experiences of medicines management in elderly, hospitalised patients in Abu Dhabi. Int J Clin Pharm. 2016;38(1):107-118. doi:10.1007/s11096-015-0212-2

59. Cullinan S, Fleming A, O'Mahony D, et al. Doctors' perspectives on the barriers to appropriate prescribing in older hospitalized patients: a qualitative study. $\mathrm{Br} J$ Clin Pharmacol. 2015;79(5):860-869. doi:10.1111/bcp. 12555

60. Manias E. Effects of interdisciplinary collaboration in hospitals on medication errors: an integrative review. Expert Opin Drug Saf. 2018;17(3):259-275. doi:10.1080/14740338.2018.1424830

61. Spinewine A, Swine C, Dhillon S, et al. Appropriateness of use of medicines in elderly inpatients: qualitative study. BMJ. 2005;331 (7522):935. doi:10.1136/bmj.38551.410012.06

62. Ferner RE, Aronson JK. Medication errors, worse than a crime. Lancet. 2000;355(9208):947-948. doi:10.1016/S0140-6736(00) 99025-1

63. McDonald J, Jayasuriya R, Harris MF. The influence of power dynamics and trust on multidisciplinary collaboration: a qualitative case study of type 2 diabetes mellitus. BMC Health Serv Res. 2012;12(1):63. doi:10.1186/1472-6963-12-63
64. Chui MA, Stone JA, Odukoya OK, Maxwell L. Facilitating collaboration between pharmacists and physicians using an iterative interview process. J Am Pharm Assoc. 2014;54(1):35-41. doi:10.1331/ JAPhA.2014.13104

65. Goyal P, Requijo T, Siceloff B, et al. Patient-reported barriers and facilitators to deprescribing cardiovascular medications. Drugs Aging. 2020;37(2):125-135. doi:10.1007/s40266-019-00729-x

66. Reeve E, Low L-F, Hilmer SN. Beliefs and attitudes of older adults and carers about deprescribing of medications: a qualitative focus group study. Br J Gen Pract. 2016;66(649):e552-e560. doi:10.3399/ bjgp16X685669

67. Zechmann S, Trueb C, Valeri F, Streit S, Senn O, Neuner-Jehle S. Barriers and enablers for deprescribing among older, multimorbid patients with polypharmacy: an explorative study from Switzerland. BMC Fam Pract. 2019;20(1):64. doi:10.1186/s12875-019-0953-4

68. Luymes CH, van der Kleij RMJJ, Poortvliet RKE, de Ruijter W, Reis $\mathrm{R}$, Numans ME. Deprescribing potentially inappropriate preventive cardiovascular medication: barriers and enablers for patients and general practitioners. Ann Pharmacother. 2016;50(6):446-454. doi:10.1177/1060028016637181

69. Vermeir P, Vandijck D, Degroote S, et al. Communication in healthcare: a narrative review of the literature and practical recommendations. Int $J$ Clin Pract. 2015;69(11):1257-1267. doi:10.1111/ ijcp. 12686

70. Weetman K, Dale J, Spencer R, Scott E, Schnurr S. GP perspectives on hospital discharge letters: an interview and focus group study. BJGP Open. 2020;4(2):bjgpopen20X101031. doi:10.3399/ bjgpopen20X101031

71. Wills M, Hyland L, Chearman T, Cross F, Verrier-Jones K. GP and hospital doctor perspectives on the hospital patient discharge process. Bull Royal Coll Surg Engl. 2011;93(1):1-4. doi:10.1308/ 147363511 X546518

72. Kripalani S, LeFevre F, Phillips CO, Williams MV, Basaviah P, Baker DW. Deficits in communication and information transfer between hospital-based and primary care physicians: implications for patient safety and continuity of care. JAMA. 2007;297(8):831-841. doi:10.1001/jama.297.8.831

73. Hill-Taylor B, Sketris I, Hayden J, Byrne S, O’Sullivan D, Christie R. Application of the STOPP/START criteria: a systematic review of the prevalence of potentially inappropriate prescribing in older adults, and evidence of clinical, humanistic and economic impact. $J$ Clin Pharm Ther. 2013;38(5):360-372. doi:10.1111/jcpt.12059

74. Ferrill MJ, Brown DA, Kyle JA. Clinical versus statistical significance: interpreting $P$ values and confidence intervals related to measures of association to guide decision making. J Pharm Pract. 2010;23(4):344-351. doi:10.1177/0897190009358774

75. Page P. Beyond statistical significance: clinical interpretation of rehabilitation research literature. Int J Sports Phys Ther. 2014;9(5):726736.
Drug, Healthcare and Patient Safety

\section{Publish your work in this journal}

Drug, Healthcare and Patient Safety is an international, peer-reviewed open-access journal exploring patient safety issues in the healthcare continuum from diagnostic and screening interventions through to treatment, drug therapy and surgery. The journal is characterized by the rapid reporting of reviews, original research, clinical, epidemiological and post-marketing surveillance studies, risk management, health literacy and educational programs across all areas of healthcare delivery. The manuscript management system is completely online and includes a very quick and fair peer-review system. Visit $\mathrm{http}: / /$ www.dovepress.com/testimonials.php to read real quotes from published authors. 